\title{
Generalized ray method for three-dimensional propagation in a penetrable wedge
}

This paper is dedicated to the memory of Franz Ziegler

Received: 24 August 2017 / Revised: 4 October 2017 / Published online: 17 January 2018

(C) The Author(s) 2018. This article is an open access publication

\begin{abstract}
A generalized ray solution is presented for the three-dimensional (3-D) acoustic field produced by a transient point source in a rigid-bottom, fast-speed fluid-bottom, and fast-speed elastic-bottom wedge modeling a continental shelf environment. The effect of diffraction (scattering) at the wedge apex is ignored in the solution, but nevertheless the solution is exact and complete for the image component of the field, expressed by a sum of partial waves including a pulse emitted from the source plus a finite number of pulses reflected off the wedge boundaries. Time records of the acoustic pressure illustrating 3-D acoustical propagation effects are evaluated at five receivers located at equal radial range in the horizontal from the source, but different orientation to the source, measured by the azimuthal angle assuming values: $0^{\circ}$ (down-slope), $45^{\circ}$ (obliquely down-slope), $90^{\circ}$ (cross-slope), $135^{\circ}$ (obliquely up-slope), and $180^{\circ}$ (up-slope). The arrival times of the spherical and head waves propagating along various paths are evaluated at each receiver for each bottom. It was found that the time interval between the first arrival and the ultimate arrival diminished, and the pulses became more peaked, as the azimuthal angle of the receiver increased. For the rigid-bottom wedge, we have found that all backscattered pulses are significant at the up-slope receiver. For the fluid-bottom wedge, we have found that: at each receiver, the source-pulse is preceded by the ground wave which is much weaker than the water wave; and, at the up-slope receiver, the backscattered pulses are insignificant. For the elastic-bottom wedge, we have found that: at each receiver, the ground-wave-response begins earlier than that in the fluid-bottom wedge, and thus the record separates out into distinct ground-wave and water-wave phases; and, at the up-slope receiver, the backscattered pulses are significant.
\end{abstract}

\section{Introduction}

In this paper, we present a generalized ray solution for the acoustic field generated by a transient point source in a wedge of fluid overlaying a sloping bottom that is assumed to be rigid, fast-speed fluid, or fast-speed elastic medium. The horizontal boundary of the wedge is a pressure-release surface. The wedge and the fluid and elastic bottoms are homogeneous (thus isovelocity) and lossless. The wedge is three-dimensional (3-D) in

Deceased: Y.-H. Pao

P. Borejko $(\bowtie)$

Department of Civil Engineering, TU Wien, Vienna, Austria

E-mail: piotr.borejko@tuwien.ac.at

C.-F. Chen

Department of Engineering Science and Ocean Engineering, National Taiwan University, Taipei, Taiwan, Republic of China

E-mail: chifang@ntu.edu.tw

Y.-H. Pao

Institute of Applied Mechanics, National Taiwan University, Taipei, Taiwan, Republic of China 
that the source and the receiver are not restricted to the plane perpendicular to the wedge apex (main vertical plane), that is, the receiver may lie cross-slope as well as down-slope or up-slope from the source. This 3-D wedge is a physical model of a continental shelf environment in which the ocean bottom has a constant slope (coastal wedge or shallow water wedge).

A wedge of fluid with a pressure-release horizontal surface and a rigid bottom (perfect wedge with mixed boundaries) is the simplest model of a coastal wedge, but it is inadequate in that it cannot account for acoustical penetration of the bottom typical for a real ocean floor. A wedge of fluid with a pressure-release horizontal surface and a penetrable bottom (penetrable wedge) is a more realistic model for a sand-bottom (the case of a fluid bottom) or a rock-bottom (the case of an elastic bottom) coastal wedge.

The total field in a wedge consists of two components: the field due to diffraction (scattering) at the wedge apex, which under certain conditions vanishes in the perfect wedge with homogeneous or mixed reflecting boundaries (Refs. [1-6]), and the image field represented by the wave emitted from the source plus (opposite to a parallel layer) a finite number of multi-reflected waves. The head (lateral) wave with the conical front is excited in a penetrable wedge whenever a multi-reflected spherical wave undergoes the total reflection at a fast-speed bottom, and the head wave front propagating along the refracted ray path arrives at a receiver ahead of the spherical wave front following the stationary time path.

Since the wedge is a nonparallel-sided waveguide, these (refracted and stationary) paths are essentially 3-D. The repeated reflections of acoustic energy at the sloping bottom introduce a curvature into the projection of the propagation path onto the horizontal surface of the wedge, provided the segment of this path originating at the source has a cross-slope component. This path curvature in the horizontal is known as the horizontal refraction (Refs. [7-12]). When such a path leaves the source propagating up-slope, it may be turned around to reach a receiver propagating down-slope. This change in propagation direction occurs as a result of multiple reflections at the sloping bottom. A receiver that is not restricted to the main vertical plane containing the source can thus be reached along one of the two paths: a direct path or an indirect path that is turned around on approaching the wedge apex. If a receiver is restricted to the main vertical plane, the change in propagation direction is referred to as the backscattering, and indirect paths are referred to as backscattered paths (Refs. $[9,13])$.

The horizontal refraction and backscattering are essential features of acoustical propagation in wedge-like waveguides. The field is then $3-\mathrm{D}$, that is, it depends on three spatial coordinates: the range (normal to the apex), the cross-range (parallel to the apex), and the depth coordinate.

Most previous work on acoustical propagation on a coastal wedge treated the perfect wedge model with homogeneous or mixed boundaries (Refs. [2-6,14-16]). The analytical solution for the field in a perfect wedge is exact, since the Helmholtz equation for this problem is separable. However, for the penetrable wedge problem, the Helmholtz equation is no longer separable, and an exact analytical solution for the field does not exist (Refs. $[4,17])$.

In shallow water acoustics, the widely used analysis of acoustical propagation from waterborne sources is that in terms of the normal-mode theory, but the theory is restricted only to stratified media with plane-parallel interfaces. Also, a sum of all the normal modes, as such, does not establish the entire field, and the branch line integral that represents the remainder should not be discarded for short-range propagation, as discussed in Refs. [18-20].

Nevertheless, the normal-mode method may be extended to a coastal wedge with a slowly sloping bottom by neglecting the coupling between normal modes due to horizontal variation of the water depth. Such an (approximately correct) analysis is known as the adiabatic mode theory (Refs. [10,21-23]), and the theory predicts that the normal modes, as they propagate, adjust their depth profiles to the local water depth, but the theory in its original form fails to explain disappearance into the underlying bottom of an up-slope propagating mode when it encounters its critical (cutoff) depth, beyond which it can no longer exist as a propagating wave (Refs. [24-26]). The validity of the assumption of uncoupled normal modes was carefully examined by conducting measurements of guided modes in model experiments (Refs. $[22,27,28]$ ) and in a real ocean (Refs. $[29,30])$, and by comparing numerical parabolic equation (PE) calculations with adiabatic mode predictions (Refs. [26,31]).

An alternate modeling approach to the normal-mode method is that in terms of the so-called complex ray method, which accurately predicts the field at short and long ranges, as well as in the region of the critical angle where a propagating mode is near cutoff and the normal-mode method may be invalid at all ranges. Such a ray method was developed early by Tindle et al. (Refs. [32-34]) for investigating acoustical propagation in a Pekeris-type environment (horizontal seabed), and later extended by Westwood (Refs. $[13,35,36])$ to model a modal structure of the field on a coastal wedge. In Ref. [35], three interesting features of 3-D acoustical 
propagation in a fluid-bottom wedge model are discussed: modal shadow zones, multiple mode arrivals, and curvature of the mode wavefronts. The first and second effects were described also by Buckingham (Ref. [17]) who derived an approximate solution of acoustic waves in a penetrable wedge, and Sturm (Ref. [37]) and Sturm and Korakas (Ref. [38]) who applied a PE method to treat acoustical propagation in a slopingbottom environment, and observed by Korakas et al. (Ref. [39]) and Sturm and Korakas (Ref. [38]) in a model experiment for cross-slope propagation. The third effect was also treated by Jensen and Tindle (Ref. [27]) who used a PE method to simulate down- and up-slope propagation, and observed by Tindle et al. (Ref. [66]) in a model experiment for down-slope propagation.

Further experimental studies of propagation effects in a wedge-shaped domain were presented in Refs. [41-44]. A visual picture of the horizontal curvature phenomenon was given in Ref. [41], but no comparison with a theoretical model was made. Experiments conducted in a wedge-like ocean (Ref. [42]) confirmed theoretical predictions of the horizontal refraction (Ref. [5]). The formation of acoustic shadow zones in cross-slope propagation, arising from the bending of the propagation paths in the horizontal, was investigated experimentally in a perfect wedge with pressure-release boundaries (Ref. [43]) and in a penetrable wedge with an elastic bottom (Ref. [44]), and the experimental results showed good agreement with the theoretical models (Refs. [5,45]).

As reviewed in Ref. [46], there has been limited application of the above-summarized modeling approaches to measured data, primarily due to the lack of experimental observation of 3-D propagation of underwater sound on a coastal wedge. Only recently the 3-D sound was quantitatively measured in a pair of acoustic transmission tests on the (wedge-like) Florida shelf (Ref. [47]), and then accurately modeled by applying a novel hybrid approach (Ref. [46]) and that based on a 3-D adiabatic mode technique (Ref. [48]), both approaches achieving excellent agreement with data.

The generalized ray theory of transient waves in layered media was primarily developed for geophysical applications. They were made to investigate the propagation of sound pulses in shallow water, which was modeled by two or three layers of fluid; the layered model was also applied to interpret seismograms of natural and artificial seismic events [see the articles by Pao and Gajewski (Ref. [49]) and Pao (Ref. [50]), and the monograph by Kennett (Ref. [51])]. The early applications of the theory were confined to parallelly layered models. For a sloping surface layer of soil overlaying a basement or a sloping-bottom continental shelf environment, the canonical model is that of a wedge-shaped medium overlaying a half-space. The theory was thus adopted by Pao and Ziegler (Ref. [52]), Ziegler and Pao (Refs. [53,54]), Ziegler et al. (Refs. [55,56]), Borejko (Ref. [57]), and Borejko and Ziegler (Ref. [58]) for analyzing elastic waves in a wedge of solid to explain the effect of a sloping surface layer of soft deposits on the local magnification of ground surface motion (earthquake intensity); and by Pao et al. (Ref. [59]) for analyzing acoustic waves in a penetrable wedge of fluid to investigate propagation characteristics of underwater sound on a coastal wedge.

In the generalized ray solution for the acoustic field in a penetrable wedge of fluid, the contribution from the diffracted (scattered) component of the field is not included. Nevertheless, the solution is exact and complete for the image component of the field, expressed by a sum of partial waves: the 0th order partial wave is that radiated from the source, and the $k$ th order partial wave $(k=1,2,3, \ldots)$ is that reflected $k$-times from the wedge boundaries before arriving at the receiver. When the wedge angle $\alpha$ is an integer submultiple of $\pi$, the number of partial waves contributing to the image field is then $1+2 \pi / \alpha$.

For transient waves, the Laplace transform with respect to time is used; and a representation of each Laplace-transformed partial wave is obtained in the form of a double integral over infinite intervals in the slowness variables, known in the generalized ray theory as a "ray integral" (Ref. [49]). The inverse Laplace transform of each ray integral is then evaluated exactly by applying the Cagniard-de Hoop method so that the integral representation of each partial wave is then reduced to the form of a single integral over a finite interval in the slowness variable, which is further transformed into the form amenable to numerical integration accomplished by the Gaussian quadrature (Ref. [59]).

The values of each ray integral, calculated at a preselected time interval, are convoluted with a time function of the source to obtain complete pressure response of the particular partial wave at a receiver point, including the contribution from the multi-reflected spherical wave, which, depending on the receiver position and the number of consecutive reflections, may be preceded by a number of contributions from the head waves (ground wave). For a fast-speed elastic bottom, the response also includes the contributions from the pseudo-Rayleigh and Scholte (Stoneley) waves. Besides the partial pressure responses, the solution computes also the travel times and propagation paths for the spherical and head waves. 


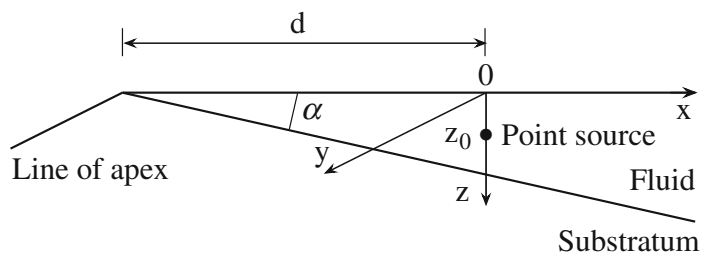

Fig. 1 Geometry of the wedge of fluid $(c=1, \rho=1)$ over the rigid, fast-speed fluid $\left(c_{p}=1.2, \rho_{2}=2\right)$, or fast-speed elastic $\left(c_{p}=\sqrt{3} c_{s}, c_{s}=2.19, \rho_{2}=2.5\right)$ bottom. The point source is located in the wedge at $\boldsymbol{x}_{\boldsymbol{o}}=\left(0,0, z_{\mathrm{o}}\right)$

On comparing the relative strengths of various partial waves, one can ascertain which contributions and mechanisms of propagation are significant and which are not, in particular, one can determine whether backscattered arrivals are significant. This is an important issue, since some numerical modeling approaches are intrinsically unable to account for backscattering. Another important practical issue is the presence of head, pseudoRayleigh, and Scholte waves in the solution, since in situ measurements of the arrivals of these waves in a sloping-bottom environment might be compared with theoretical simulations to determine the geoacoustic properties of the bottom.

As mentioned above, Pao et al. (Ref. [59]) developed the generalized ray solution for acoustical signals emitted from a point source in a penetrable wedge. However, their solution was applicable to the case of a slow-speed fluid bottom and a fast-speed fluid bottom only. Hence, Borejko et al. (Ref. [60]) extended the solution given in Pao et al. (Ref. [59]) to treat also the case of a fast-speed elastic bottom, but they did not evaluate the complete acoustical signal recorded by a receiver.

In this paper, the solution given in Borejko et al. (Ref. [60]) is further extended to evaluate the complete time records of received acoustical signals including the contribution of the entire acoustic field (with the omission of the effect of diffraction at the wedge apex) at the locations down-, cross-, and up-slope at equal radial range in the horizontal from the point source in the rigid-bottom, fast-speed fluid-bottom, and fast-speed elastic-bottom wedge, thus illustrating 3-D acoustical propagation effects in the penetrable wedge of fluid. The solution is also supplemented by a detailed discussion of evaluation of the ray integrals for partial wave motions.

The paper is organized as follows: In Sect. 2 we define the geometry of the wedge of fluid, summarize the governing equations for the field, and recall the boundary conditions for the perfect and penetrable wedge. Section 3 presents a brief discussion of the field, including decomposition of the image field, mechanism of excitation of the head waves, and phenomena of horizontal refraction and backscattering. In Sect. 4 the generalized ray solution for the image field for the case of a fast-speed elastic bottom is summarized, in Sect. 5 numerical examples of complete time records of received acoustical signals are given, and our conclusions are presented in Sect. 6.

\section{Mathematical formulation of the problem}

The geometry for the present wedge problem is illustrated in Fig. 1. A wedge-shaped layer of fluid, formed by two inclined planes that intersect along the line of apex, overlies a bottom that may be a rigid, fluid, or elastic medium. The apex angle (wedge angle) is $\alpha$, and it may lie anywhere between 0 and $\pi / 2$.

Two Cartesian coordinates systems $(x, y, z)$ and $\left(x^{\prime}, y^{\prime}, z^{\prime}\right)$ are employed in the analysis. The $x, y$, and $z$ are the range (normal to the apex), the cross-range (parallel to the apex), and the depth coordinate axes, respectively. The $x^{\prime}$ and $z^{\prime}$ axes are obtained by rotating the $x$ and $z$ axes, respectively, through the angle $\alpha$ about the $y$ axis. The common origin 0 of the two coordinate systems is located at the horizontal boundary plane of the wedge $z=0$, and its range from the apex line is $d$.

The point source is situated in the plane $y=0$ (main vertical plane) within the wedge at a depth $z_{\mathrm{o}}$ directly below the origin 0 , so that its range from the apex line is also $d$, and its position vector is $\boldsymbol{x}_{\mathrm{o}}=\left(0,0, z_{\mathrm{o}}\right)$ in the unprimed system. The sloping interface separating the wedge from the bottom is defined by $z^{\prime}=h \cos \alpha$, where $h$ is the fluid depth measured vertically at the source location, thus $d=h \cos \alpha / \sin \alpha$. Our task is to derive and analyze the field at any point receiver situated within the wedge. 


\subsection{The governing equations}

In a wedge of fluid, the field is specified by the velocity potential function $\phi(x, t)$, which satisfies the quiescent initial conditions

$$
\phi=0, \quad \frac{\partial \phi}{\partial t}=0, \quad \text { at } t=0,
$$

and the 3-D inhomogeneous wave equation,

$$
c^{2} \nabla^{2} \phi-\frac{\partial^{2} \phi}{\partial t^{2}}=-f(t) \delta(x) \delta(y) \delta\left(z-z_{\mathrm{o}}\right)
$$

The $c$ is the sound speed in the wedge, and the causal function $f(t)$ (that is, $f(t)=0$ for $t<0$ ) specifies the time history of $\phi$ at the source. The excess acoustic pressure $p(x, t)$ above the ambient pressure and the velocity $\boldsymbol{v}(\boldsymbol{x}, t)$ are given by

$$
p=-\rho \frac{\partial \phi}{\partial t}, \quad v=\nabla \phi,
$$

respectively, where $\rho$ is the density. It is assumed that the fluid in the wedge is homogeneous, that is, $c$ and $\rho$ are independent of the position.

In a fluid bottom of density $\rho_{2}$, the wave field is defined by the velocity potential function $\phi_{2}(\boldsymbol{x}, t)$, which satisfies the 3-D homogeneous wave equation,

$$
c_{p}^{2} \nabla^{2} \phi_{2}-\frac{\partial^{2} \phi_{2}}{\partial t^{2}}=0,
$$

where $c_{p}$ is the sound speed. The pressure $p_{2}(\boldsymbol{x}, t)$ and the velocity $\boldsymbol{v}_{2}(\boldsymbol{x}, t)$ are $p_{2}=-\rho_{2}\left(\partial \phi_{2} / \partial t\right)$ and $\boldsymbol{v}_{2}=\nabla \phi_{2}$, respectively.

In an elastic bottom having a density $\rho_{2}$, the wave field is characterized by the displacement vector $\boldsymbol{u}(\boldsymbol{x}, t)$, which satisfies the Navier-Cauchy equation of motion,

$$
\left(c_{p}^{2}-c_{s}^{2}\right) \nabla \nabla \cdot \boldsymbol{u}+c_{s}^{2} \nabla^{2} \boldsymbol{u}-\frac{\partial^{2} \boldsymbol{u}}{\partial t^{2}}=0,
$$

where $c_{p}$ and $c_{s}$ are the $P$ and $S$ wave speeds, respectively, and the external body forces are supposed to be absent. The stress tensor $\boldsymbol{\sigma}(\boldsymbol{x}, t)$ in an elastic bottom is related to the displacement gradient $\nabla \boldsymbol{u}(\boldsymbol{x}, t)$ by Hooke's law for an isotropic elastic solid.

It is assumed that both the fluid and elastic bottoms are homogeneous, that is, $c_{p}, c_{s}$, and $\rho_{2}$ are independent of the position.

\subsection{The boundary conditions}

The horizontal surface of the wedge $z=0$ is pressure-release (Dirichlet boundary) so that the boundary condition for $p(\boldsymbol{x}, t)$ is

$$
p=0 \text { at } z=0
$$

Since the bottom is assumed to be a rigid, fluid, or elastic medium, there are three different sets of boundary conditions at the sloping interface $z^{\prime}=h \cos \alpha$.

Case A Rigid sloping interface (Neumann boundary) At the Neumann boundary, the normal derivative of the velocity potential function is zero,

$$
\frac{\partial \phi}{\partial z^{\prime}}=0, \quad \text { at } \quad z^{\prime}=h \cos \alpha .
$$

Case B Fluid-fluid sloping interface For the case of a fluid bottom, the boundary conditions are

$$
p=p_{2}, \quad v_{z}^{\prime}=v_{2 z}^{\prime} \quad \text { at } \quad z^{\prime}=h \cos \alpha,
$$

where the first and second conditions ensure the continuity of pressure and normal velocity, respectively. 
Case C Fluid-elastic solid sloping interface For the case of an elastic bottom, the boundary conditions are

$$
p=-\sigma_{z z}^{\prime}, \quad v_{z}^{\prime}=\frac{\partial u_{z}^{\prime}}{\partial t}, \quad \sigma_{z x}^{\prime}=\sigma_{z y}^{\prime}=0 \quad \text { at } \quad z^{\prime}=h \cos \alpha
$$

where the first and second conditions ensure the continuity of normal stress and normal velocity, respectively, and the third condition ensures the vanishing of shearing stresses. The tangential components of the velocity vectors in the two adjacent media, that is, in case B, fluid and fluid, and, in case C, fluid and elastic solid, are discontinuous at the sloping interface $z^{\prime}=h \cos \alpha$.

\section{Acoustic field in a wedge of fluid}

In general, the field in a wedge of fluid includes two components: the diffracted field due to scattering at the wedge apex and the image field, which can be expressed by a sum of partial waves including the wave emitted by the source $\phi_{0}(\boldsymbol{x}, t)$ and a number of multi-reflected waves $\phi_{ \pm k}(\boldsymbol{x}, t)(k=1,2,3, \ldots)$ arising from the constraints imposed by the wedge boundaries on the field. In $\phi_{ \pm k}$, the $k$ letter indicates the number of reflections from the boundaries, the + sign indicates that the first reflection is from the sloping boundary $z^{\prime}=h \cos \alpha$, and the - sign indicates that the first reflection is from the horizontal boundary $z=0$.

\subsection{Decomposition of the image field}

The diffracted field component is ignored in the following discussion, and the field is thus given by the image field component of the form

$$
\phi=\phi_{\mathrm{o}}+\phi_{ \pm 1}+\phi_{ \pm 2}+\phi_{ \pm 3}+\cdots .
$$

The $\phi_{+k}$ is the $+k$ th partial wave that undergoes $k$ consecutive reflections from the wedge boundaries, the first reflection being from the sloping boundary $z^{\prime}=h \cos \alpha ; \phi_{-k}$ is the $-k$ th partial wave that undergoes $k$ consecutive reflections from the wedge boundaries, the first reflection being from the horizontal boundary $z=0$.

The $\phi_{0}$, which is the particular solution of the inhomogeneous wave equation (2.2) subject to the initial conditions (2.1) is represented by the spherical $P$ wave of the form

$$
\phi_{\mathrm{o}}(\boldsymbol{x}, t)=f(t-R / c) / 4 \pi c^{2} R,
$$

as was shown in Ref. [61], where $R^{2}=x^{2}+y^{2}+\left(z-z_{\mathrm{o}}\right)^{2}$.

\subsection{Propagation of the spherical and head waves in a wedge of fluid}

Between the source and the receiver, there is a minimum travel-time path for the $\pm k$ th multi-reflected spherical $P$ wave, known as the $\pm k$ th stationary time path ( $\pm k$ th ray path), the broken line connecting the source with the receiver via successive reflection points. The ray paths in a wedge of fluid can be constructed geometrically by the method of images.

A head $P$ wave with the conical front is generated in a wedge of fluid when the angle of incidence of the spherical $P$ wave, that is incident from the wedge upon a fast-speed bottom, exceeds the critical angle, that is, when the spherical $P$ wave is totally reflected from a fast-speed bottom (cf. Refs. [18,62-64]). Specifically, for a fast-speed elastic bottom $\left(c_{p}>c_{s}>c\right)$, two head $P$ waves are generated in the wedge when $\gamma>\gamma_{c s}>\gamma_{c p}$, where $\gamma$ is the angle of incidence, and $\gamma_{c s}=\sin ^{-1}\left(c / c_{s}\right)$ and $\gamma_{c p}=\sin ^{-1}\left(c / c_{p}\right)$ are the critical angles. For a fast-speed fluid bottom $\left(c_{p}>c\right)$, one head $P$ wave is generated in the wedge when $\gamma>\gamma_{c p}$. The head wave fronts propagating along the refracted ray paths arrive at the receiver ahead of the spherical wave fronts following the stationary time paths. The refracted ray paths in a wedge of fluid can be constructed geometrically by the method of images.

In the wedge, the local angle of incidence of the $\pm k$ th multi-reflected spherical $P$ wave changes from one reflection to the next. On successive reflections from either one of the wedge boundaries, this angle decreases by twice the apex angle for up-slope propagation and increases by twice the apex angle for down-slope propagation. The $\pm k$ th spherical $P$ wave may thus strike the bottom either at steep angles (smaller than the 
critical angle), where partial reflection occurs with energy loss into the bottom, or at shallow angles (larger than the critical angle), where total reflection occurs with no energy loss. The head $P$ waves are generated by these bottom reflections, where the local angle of incidence exceeds the critical angle. The multiple bottom interactions of the $\pm k$ th spherical $P$ wave may thus initiate a large number of the head $P$ waves, arriving at the receiver ahead of the $\pm k$ th spherical $P$ wave (cf. Refs. $[13,36]$ ). The head $P$ wave that arrives first at the receiver ( $\pm k$ th primary head $P$ wave) is always initiated either by the first bottom reflection or the ultimate bottom reflection of the $\pm k$ th multi-reflected spherical $P$ wave. The head $P$ waves that follow the $\pm k$ th primary head $P$ wave are referred to as the secondary head $P$ waves.

Since a wedge of fluid is a nonparallel-sided waveguide, the stationary time paths and the refracted ray paths are essentially 3 -D. When the first reflection point is out of the main vertical plane $y=0$ containing the source, the repeated reflections at the sloping bottom introduce a curvature into the projection of the respective path onto the horizontal surface $z=0$, because the local plane of incidence is not a vertical plane for each bottom reflection. This path curvature in the horizontal is known as the horizontal refraction (Refs. [7-12]). Note that the whole path is in the main vertical plane $y=0$ when the first reflection point is in this plane.

When the first reflection point is located in the main vertical plane $y=0$ up-slope from the source, then, in the course of successive up-slope reflections, the local angle of incidence may become so small that the path is turned around and the wave front (spherical $P$ wave front or the head $P$ wave front) proceeds to travel down-slope. This change in the propagation direction in the main vertical plane $y=0$ is known as the backscattering (Ref. [9]). Depending on the number of reflections $k$, the respective wave front can thus reach a down-slope/up-slope receiver along one of the two paths: the direct path or the backscattered (indirect) path that is turned around on approaching the wedge apex.

An example of the minimum travel-time paths for the head $P$ waves and the spherical $P$ wave in a wedge of fluid with a fast-speed elastic bottom is given in Ref. [60, Fig. 5.1], where the two head $P$ waves arrive at the receiver along the refracted ray paths, the spherical $P$ wave arrives at the receiver along the stationary time path, and all three paths are horizontally refracted, since the receiver is placed out of the main vertical plane $y=0$ containing the source.

\section{Solution for the acoustic field in a wedge of fluid}

In this Section, we briefly summarize the generalized ray solution for the image field in a wedge of fluid. As shown in Refs. $[49,59,60]$, one can construct the exact integral representation (ray integral) for the Laplacetransformed $\pm k$ th partial wave by superposition of Laplace-transformed plane wave components traveling along the $\pm k$ th ray path, composed of $k+1$ segments. The segments, the local slowness vectors of plane wave components along each segment, and the reflection points are numbered consecutively using the index $l(l=0,1,2,3, \ldots, k)$, so that the index 0 indicates the segment originating at the source and the local slowness vector along it, the index 1 indicates the segment originating at the first reflection point and the local segment along it, and so forth.

\subsection{Exact integral representation for the multi-reflected wave motion}

Omitting all details, we only recall here that, for each reflection from the horizontal or sloping boundary: the amplitudes of the plane wave components are altered by the corresponding reflection coefficient, the tangential components of the local slowness vectors along the incident and reflected segments are the same, and the directions of the normal components are opposite (Snell's law of reflection). The phase function $g_{ \pm k}$ of the plane wave component of the $\pm k$ th partial wave can be derived either by evaluating the total travel time along the $\pm k$ th ray path or by the method of images, and it is given by (Ref. [60])

$$
g_{ \pm k}=-\xi_{ \pm l}^{\prime} \cdot \boldsymbol{x}_{ \pm l}^{\prime}
$$

where the dot denotes the scalar product. In $\boldsymbol{x}_{ \pm l}^{\prime}=\left(x_{ \pm l}^{\prime}, y_{ \pm l}^{\prime}, z_{ \pm l}^{\prime}\right), x_{ \pm l}^{\prime}, y_{ \pm l}^{\prime}$, and $z_{ \pm l}^{\prime}$ are referred to as the equivalent distances between the source and the receiver along the $x^{\prime}, y^{\prime}$, and $z^{\prime}$ axes, respectively, since the 3 -D zigzag $\pm k$ th ray path can be identified as a fictitious straight ray connecting one image of the source with one image of the receiver. The $\xi_{ \pm l}^{\prime}=\left[-i \xi_{ \pm l}^{\prime},-i \eta_{ \pm l}^{\prime},(-1)^{l} \varepsilon^{\prime} \zeta_{ \pm l}^{\prime}\right]$ is the local slowness vector along the $l$ th segment of the $\pm k$ th ray path, where $i^{2}=-1 ; \zeta_{ \pm l}^{\prime}=\left(a^{2}+\xi_{ \pm l}^{\prime 2}+\eta_{ \pm l}^{\prime 2}\right)^{1 / 2}$, and $a=1 / c$ is the slowness in the 
wedge of fluid; $\varepsilon^{\prime}=+1$ and $l=0,1,2,3, \ldots, k$ for the $+k$ th ray path; and $\varepsilon^{\prime}=-1$ and $l=1,2,3, \ldots, k$ for the $-k$ th ray path.

The Laplace-transformed $\pm k$ th partial wave is thus given by the ray integral of the form

$$
\bar{\phi}_{ \pm k}(s)=\frac{s \bar{f}(s)}{8 \pi^{2} c^{2}} \int_{-\infty}^{\infty} \int_{-\infty}^{\infty} S \Pi_{ \pm k} \exp \left(s g_{ \pm k}\right) \mathrm{d} \xi_{ \pm l}^{\prime} \mathrm{d} \eta_{ \pm l}^{\prime}
$$

where the bar denotes the Laplace transform in time $t$, with transform parameter $s, S$ is the source function, and $\Pi_{ \pm k}$ is the cumulative product of consecutive reflection coefficients. The derivation of the ray integral in (4.2) is described more fully in Ref. [60].

At the pressure-release and rigid surfaces (perfectly reflecting surfaces), the reflection coefficients are $R=$ -1 and $R=1$, respectively, and the product $\Pi_{ \pm k}$ is thus constant for a wedge of fluid with perfectly reflecting boundaries. For the fluid-fluid and fluid-elastic solid sloping interfaces (penetrable bottoms), the reflection coefficients are expressed in terms of the slowness vector of the locally incident plane wave component, and are given in Refs. [59,60].

The sum of a number of ray integrals,

$$
\bar{\phi}_{\mathrm{o}}+\bar{\phi}_{ \pm 1}+\bar{\phi}_{ \pm 2}+\cdots
$$

representing the image field $\bar{\phi}$ in a wedge of fluid, satisfies the boundary conditions (2.4) and (2.5) (Ref. [60]).

\subsection{Transformation of the ray integral into the time domain}

The ray integral in (4.2) can be transformed into the time domain by applying the Cagniard-de Hoop method, as was shown in Ref. [60]. Here, we only summarize the key steps of this transformation.

The variables of integration in (4.2) are changed from $\left(\xi_{ \pm l}^{\prime}, \eta_{ \pm l}^{\prime}\right)$ to $\left(p^{\prime}, q^{\prime}\right)$ :

$$
\xi_{ \pm l}^{\prime}=p^{\prime} \cos \theta_{ \pm l}^{\prime}-q^{\prime} \sin \theta_{ \pm l}^{\prime}, \quad \eta_{ \pm l}^{\prime}=p^{\prime} \sin \theta_{ \pm l}^{\prime}+q^{\prime} \cos \theta_{ \pm l}^{\prime}
$$

where $\sin \theta_{ \pm l}^{\prime}=y_{ \pm l}^{\prime} / r_{ \pm l}^{\prime}, \cos \theta_{ \pm l}^{\prime}=x_{ \pm l}^{\prime} / r_{ \pm l}^{\prime}$, and $r_{ \pm l}^{\prime 2}=x_{ \pm l}^{\prime 2}+y_{ \pm l}^{\prime 2}$. The phase function $g_{ \pm k}$ in (4.1) is thus rewritten as

$$
g_{ \pm k}\left(p^{\prime}, q^{\prime}\right)=i p^{\prime} r_{ \pm l}^{\prime}-z_{ \pm l}^{\prime}\left[a^{2}+p^{\prime 2}+q^{\prime^{2}}\right]^{1 / 2}
$$

Subsequently, the prime attached to $p$ and $q$ will be omitted. The integration variable $p$ is further transformed to a new variable $t$, defined by the phase function $g_{ \pm k}$ in (4.3), that is,

$$
-t=g_{ \pm k}(p, q) \text {. }
$$

In the above $q$ is held as a real parameter, $p$ is allowed to be complex, and $t$ has the dimension of time.

The integrand of the ray integral has the branch points corresponding to the wave speeds $c, c_{p}$, and $c_{s}$. To make it a single-valued function of $p$ in the complex $p$-plane, we introduce some branch cuts. The $p$-integration, which is now along the half-axis $\operatorname{Re} p \geq 0$, is equivalent to that along the Cagniard-de Hoop contour in the complex $p$-plane, parameterized by $t$, and defined as $p=g_{ \pm k}^{-1}(t, q)$, the inverse transformation of (4.4). Interchanging the order of integration and assuming $f\left(0^{+}\right)=0$, we then find the inverse Laplace transform of the ray integral by inspection,

$$
\phi_{ \pm k}(t)=\int_{0}^{t} \dot{f}(t-\tau) I_{ \pm k}(\tau) \mathrm{d} \tau
$$

where a dot denotes the time differentiation, and

$$
I_{ \pm k}(t)=\frac{a^{2}}{2 \pi^{2}} H\left(t-t_{A}\right) \operatorname{Re} \int_{0}^{q(t)} S \Pi_{ \pm k} \frac{\mathrm{d} g_{ \pm k}^{-1}}{\mathrm{~d} t} \mathrm{~d} q .
$$

In the above, $H(t)$ is the Heaviside step function, $t_{A}=a z_{ \pm l}^{\prime}$, Re denotes the real part, the upper limit for the $q$-integration is $q(t)=\left[\left(t / z_{ \pm l}^{\prime}\right)^{2}-a^{2}\right]^{1 / 2}$, and

$$
\frac{\mathrm{d} g_{ \pm k}^{-1}}{\mathrm{~d} t}=\frac{i\left[a^{2}+p^{2}+q^{2}\right]^{1 / 2}}{\left[\left(a^{2}+q^{2}\right)\left(r_{ \pm l}^{\prime 2}+z_{ \pm l}^{\prime 2}\right)-t^{2}\right]^{1 / 2}} .
$$


The ray integral $I_{ \pm k}$ in (4.5) is exact, and thus it represents the exact time domain response of the entire wave motion due to the $\phi_{ \pm k}$ partial wave.

For a perfect wedge of fluid with the mixed Dirichlet-Neumann boundaries (2.4) and (2.5.1), the product $\Pi_{ \pm k}$ is constant, and the transformation of the ray integral in (4.2) into the time domain is greatly simplified,

$$
\phi_{ \pm k}(t)=(-1)^{n} f\left(t-R_{ \pm l}^{\prime} / c\right) / 4 \pi c^{2} R_{ \pm l}^{\prime},
$$

[cf. Eq. (3.2)] where $n$ is the number of reflections from the pressure-release surface $z=0, R_{ \pm l}^{\prime 2}=r_{ \pm l}^{\prime 2}+z_{ \pm l}^{\prime 2}$, and $R_{ \pm l}^{\prime}$ is the "equivalent distance" between the source and the receiver for the $\pm k$ th ray path.

Assuming $\dot{f}\left(0^{+}\right)=0$, the partial pressure response $p_{ \pm k}(\boldsymbol{x}, t)$ of the $\pm k$ th wave is

$$
p_{ \pm k}(t)=-\rho \int_{0}^{t} \ddot{f}(t-\tau) I_{ \pm k}(\tau) \mathrm{d} \tau .
$$

\subsection{Arrival times for the spherical and head waves}

The arrival time $t_{M}$ of the $\pm k$ th multi-reflected spherical $P$ wave propagating along the $\pm k$ th ray path is determined from those values of $p$ and $q$ that make $t$ in (4.4) maximal, and it is given by (Ref. [60])

$$
t_{M}=a\left(r_{ \pm l}^{\prime 2}+z_{ \pm l}^{\prime 2}\right)^{1 / 2}
$$

In a wedge of fluid, the $\pm k$ th multi-reflected spherical $P$ wave undergoes a series of bottom reflections before it reaches the receiver. The head $P$ wave is initiated at that instant in the wedge by the particular bottom reflection, when the tangential component of the slowness vector of the spherical $P$ wave impinging on the sloping interface is equal to one of the slownesses in the fast-speed bottom $a_{p}$ or $a_{s}$, that is,

$$
\left(-i \xi_{ \pm l}^{\prime}\right)^{2}+\left(-i \eta_{ \pm l}^{\prime}\right)^{2}=a_{f}^{2} \quad f=p, s
$$

where $a_{p}=1 / c_{p}$ and $a_{s}=1 / c_{s}$. The arrival time $t_{ \pm l f}$ of the head $P$ wave propagating along the refracted ray path is then given by (Ref. [60])

$$
t_{ \pm l f}=a_{f} r_{ \pm l}^{\prime}+z_{ \pm l}^{\prime}\left(a^{2}-a_{f}^{2}\right)^{1 / 2} \quad f=p, s .
$$

For a fast-speed elastic bottom, two head $P$ waves are initiated, and $t_{ \pm l p}$ and $t_{ \pm l s}$ are the arrival times of the faster and slower waves, respectively. For a fast-speed fluid bottom, one head $P$ wave is initiated, and it arrives at the receiver at time $t=t_{ \pm l p}$.

\subsection{Evaluation of the ray integral}

The evaluation of the ray integral in (4.5) depends on the receiver location $x$ and the time of observation as compared with the critical times $t_{A}, t_{M}$, and $t_{ \pm l p} \quad\left(t_{A}<t_{ \pm l p}<t_{M}\right)$. For $t_{A} \leq t<t_{M}$, the integrand of the $q$-integral in (4.5) is nonsingular. For $t \geq t_{M}$, the $q$-integral in (4.5) contains a singularity, since $d g_{ \pm k}^{-1} / d t$ in (4.6) is singular of half-order at $q=q_{M}(t)$, where

$$
q_{M}(t)=\left[t^{2} /\left(r_{ \pm l}^{\prime 2}+z_{ \pm l}^{\prime 2}\right)-a^{2}\right]^{1 / 2}
$$

and $0<q_{M}(t)<q(t)$ when $t \geq t_{M}$.

In order to ascertain if at the specific location $x$ the arrival of the $\pm k$ th multi-reflected spherical $P$ wave is preceded by the arrival of the $\pm k$ th primary head $P$ wave, we examine the $\pm k$ th ray path pertinent to the $\pm k$ th spherical $P$ wave. There are no head wave arrivals if the tangential component of the slowness vector along each segment of the $\pm k$ th ray path, that is incident upon the sloping bottom, is less than the $a_{p}$ slowness in the fast-speed bottom. The arrival of the $\pm k$ th spherical $P$ wave at $t=t_{M}$ is preceded by the arrival of the $\pm k$ th primary head $P$ wave at $t=t_{ \pm l p}$, if for the first or the ultimate bottom reflection the tangential component of the slowness vector along the incident segment of the $\pm k$ th ray path exceeds $a_{p}$. (If for both the first and the ultimate bottom reflections the tangential component of the slowness vector along the incident segment of the $\pm k$ th ray path exceeds $a_{p}$, the $\pm k$ th primary head $P$ wave is initiated by this reflection where 
the tangential component of the slowness vector is larger.) If additionally, for other than the first or the ultimate bottom reflections, the tangential component of the slowness vector along the incident segment of the $\pm k$ th ray path exceeds $a_{p}$, the secondary head $P$ waves are initiated by these reflections, and they arrive prior to the $\pm k$ th spherical $P$ wave. Thus we consider two cases.

Case A The response begins with the arrival of the spherical $P$ wave at $t=t_{M}$, and there are no head wave arrivals. The integral in (4.5) is vanishing for $t_{A} \leq t<t_{M}$, since the integrand is an imaginary function of $q$ for $0<q<q(t)$ when $t_{A} \leq t<t_{M}$. For $t \geq t_{M}$, the integration in (4.5) may be divided into two parts, the first from 0 to $q_{M}(t)$ and the second from $q_{M}(t)$ to $q(t)$. The second integration vanishes, because the integrand is an imaginary function of $q$ for $q_{M}(t)<q<q(t)$ when $t \geq t_{M}$. Thus, in case A, only the first part of integration from 0 to $q_{M}(t)$ contributes to the response when $t \geq t_{M}$, and the $q$-integral contains a half-order singularity at the upper limit $q_{M}(t)$.

Case B The response begins with the arrival of the $\pm k$ th primary head $P$ wave at $t=t_{ \pm l p}$. In the case of an elastic bottom, this wave is referred to as the $\pm k$ th faster primary head $P$ wave, because it is followed by the $\pm k$ th slower primary head $P$ wave arriving at $t=t_{ \pm l s}$. The primary arrival at $t=t_{ \pm l p}$ may then be followed by the arrivals of the secondary head $P$ waves initiated by other than the first or the ultimate bottom reflection of the $\pm k$ th spherical $P$ wave. Finally, the $\pm k$ th spherical $P$ wave reaches the receiver at $t=t_{M}$.

The integral in (4.5) is vanishing for $t_{A} \leq t<t_{ \pm l p}$, since the integrand is an imaginary function of $q$ for $0<q<q(t)$ when $t_{A} \leq t<t_{ \pm l p}$. For $t_{ \pm l p} \leq t<t_{M}$, the integration in (4.5) may be divided into two parts, the first from 0 to $q_{p}(t)$ and the second from $q_{p}(t)$ to $q(t)$ where

$$
q_{p}(t)=\left[\left(1 / z_{ \pm l}^{\prime}\right)^{2}\left(t-a_{p} r_{ \pm l}^{\prime}\right)^{2}+a_{p}^{2}-a^{2}\right]^{1 / 2}
$$

and $0<q_{p}(t)<q(t)$. The second integration vanishes, because the integrand is an imaginary function of $q$ for $q_{p}(t)<q<q(t)$ when $t_{ \pm l p} \leq t<t_{M}$. Thus, in case B, only the first part of integration from 0 to $q_{p}(t)$ contributes to the response when $t_{ \pm l p} \leq t<t_{M}$, and the $q$-integral is nonsingular.

Since $0<q_{M}(t)<q_{p}(t)<q(t)$ when $t \geq t_{M}$, the integration in (4.5) may be divided into three parts for $t \geq t_{M}$ : The first from 0 to $q_{M}(t)$, the second from $q_{M}(t)$ to $q_{p}(t)$, and the third from $q_{p}(t)$ to $q(t)$. The third integration vanishes, because the integrand is an imaginary function of $q$ for $q_{p}(t)<q<q_{p}(t)$ when $t \geq t_{M}$. Thus, in case $\mathrm{B}$, only the first and second parts of integration from 0 to $q_{M}(t)$ and from $q_{M}(t)$ to $q(t)$ contribute to the response when $t \geq t_{M}$, and the first and second $q$-integrals contain a half-order singularity at the upper and lower limits, respectively.

In both cases, this singularity in the $q$-integrals can be removed by changing the integration variable from $q$ to $u$ :

$$
u=\left[\left(a^{2}+q^{2}\right)\left(r_{ \pm l}^{\prime 2}+z_{ \pm l}^{\prime 2}\right)-t^{2}\right]^{1 / 2}, \quad d q=\frac{\left[\left(a^{2}+q^{2}\right)\left(r_{ \pm l}^{\prime 2}+z_{ \pm l}^{\prime 2}\right)-t^{2}\right]^{1 / 2}}{q\left(r_{ \pm l}^{\prime 2}+z_{ \pm l}^{\prime 2}\right)} d u
$$

Completing this change of variable introduces a factor $\left[\left(a^{2}+q^{2}\right)\left(r_{ \pm l}^{\prime 2}+z_{ \pm l}^{\prime 2}\right)-t^{2}\right]^{1 / 2}$ in the numerator, which matches the half-order singularity of $d g_{ \pm k}^{-1} / d t$ in the denominator [(Eq. (4.6)]. The $q$-integrals are now suitable for the numerical integration which is performed by Gaussian quadrature (quadrature with controllable error), and the convolution integral in (4.8) is then evaluated numerically using Simpson's rule.

\section{Numerical examples}

When the wedge angle $\alpha$ is an integer submultiple of $\pi$, the pressure $p(x, t)$ due to the image field at the receiver point $\boldsymbol{x}$ is

$$
p=p_{\mathrm{o}}+\sum_{k=1}^{N} p_{+k}+\sum_{k=1}^{N} p_{-k}
$$

where $p_{\mathrm{o}}$ and $p_{ \pm k}$ are the partial pressure pulses due to the $\phi_{\mathrm{o}}$ and $\phi_{ \pm k}$ waves, respectively, and $N=\pi / \alpha$.

All physical quantities having the dimension of length are normalized by $h$, the thickness of the wedge measured vertically at the source location, all quantities having the dimension of time are normalized by the characteristic time $t_{c}$, where $t_{c}=h / c$, and each partial pressure pulse is normalized by a constant $p_{c}$, which 
is specified as follows. In (2.2) we set $f(t)=t H(t)$, and since $p_{\mathrm{o}}=-\rho \partial \phi_{\mathrm{o}} / \partial t$ and $\phi_{\mathrm{o}}$ is given by (3.2), the time history of the pulse emitted by the source is a Heaviside step function (a Heaviside unit source-pulse),

$$
p_{\mathrm{o}}=\left(p_{c} / R\right) H(t-R / c),
$$

where $p_{c}=-\rho / 4 \pi c^{2}$, so that the normalized pressure $p_{\mathrm{o}} / p_{c}$ due to the source itself equals unity at $R / h=1$.

Time records of the pressure due to the image field [Eq. (5.1)] were evaluated at receivers lying down-slope, cross-slope, and up-slope from the source for the following three wedges: the perfect wedge of fluid, subject to the mixed Dirichlet-Neumann boundary conditions (2.4) and (2.5.1); the penetrable wedge of fluid with a fluid bottom, subject to the boundary conditions (2.4) and (2.5.2); and the penetrable wedge of fluid with an elastic bottom, subject to the boundary conditions (2.4) and (2.5.3). The apex angle of each wedge is chosen to be the same $\alpha=10^{\circ}$, so that in (5.1) $N=18$, and 37 partial waves contribute to the image field.

The fluid in each wedge is chosen to be the same, and we assume that $c=1$ and $\rho=1$. The properties assumed for a fluid bottom are $c_{p}=1.2$ and $\rho_{2}=2$, and those assumed for an elastic bottom are $c_{s}=2.19$, $c_{p}=\sqrt{3} c_{s}$, and $\rho_{2}=2.5$. The critical angle for the fluid bottom is $\gamma_{c p}=56.44^{\circ}$, and those for the elastic bottom are $\gamma_{c s}=27.17^{\circ}$ and $\gamma_{c p}=15.29^{\circ}$. We thus consider the fast-speed fluid and elastic bottoms with discontinuities at the sloping interface both in the velocity and in the density.

In each of the three wedges, the source and the receivers are located on the bisecting plane of the wedge. Thus, the source depth is $z_{\mathrm{o}}=d \tan (\alpha / 2)$, and the coordinates of each receiver are

$$
x=r \cos \gamma, \quad y=r \sin \gamma, \quad z=(d+r \cos \gamma) \tan (\alpha / 2) .
$$

In the above $r$ is the radial range in the horizontal plane $z=0$, and $\gamma$ is the azimuthal angle measured clockwise in this plane from the $x$ axis (Fig. 1). The normalized range of the source from the apex line is $d / h=\cot 10^{\circ}$, and the normalized source depth is $z_{\mathrm{o}} / h=\cot 10^{\circ} \tan 5^{\circ}$ for $\alpha=10^{\circ}$. The thickness of each wedge measured vertically at the source location is chosen to be the same, $h=1$. We select five receivers having the same radial range, but different azimuthal angles. In (5.3) we thus assume that $\alpha=10^{\circ}, r=5 h$, and $\gamma=0^{\circ}, 45^{\circ}, 90^{\circ}, 135^{\circ}, 180^{\circ}$ for each wedge, and five receiver positions are:

$$
\begin{array}{ll}
\boldsymbol{x}_{1}=\left[5,0,\left(\cot 10^{\circ}+5\right) \tan 5^{\circ}\right], & \gamma_{1}=0^{\circ}, \\
\boldsymbol{x}_{2}=\left[5 \cos 45^{\circ}, 5 \sin 45^{\circ},\left(\cot 10^{\circ}+5 \cos 45^{\circ}\right) \tan 5^{\circ}\right], & \gamma_{2}=45^{\circ}, \\
\boldsymbol{x}_{3}=\left(0,5, \cot 10^{\circ} \tan 5^{\circ}\right), & \gamma_{3}=90^{\circ}, \\
\boldsymbol{x}_{4}=\left[-5 \sin 45^{\circ}, 5 \cos 45^{\circ},\left(\cot 10^{\circ}-5 \sin 45^{\circ}\right) \tan 5^{\circ}\right], & \gamma_{4}=135^{\circ}, \\
\boldsymbol{x}_{5}=\left[-5,0,\left(\cot 10^{\circ}-5\right) \tan 5^{\circ}\right], & \gamma_{5}=180^{\circ}
\end{array}
$$

where the components of $\boldsymbol{x}_{1}, \boldsymbol{x}_{2}$, etc. are normalized by $h$. Relative to the source, $\boldsymbol{x}_{1}$ and $\boldsymbol{x}_{2}$ are the down-slope positions, $\boldsymbol{x}_{3}$ is the cross-slope position, and $\boldsymbol{x}_{4}$ and $\boldsymbol{x}_{5}$ are the up-slope positions.

The time records of the pressure due to the image field [Eq. (5.1), $N=18$ ], which comprises contributions from 37 partial waves, were evaluated at five locations (5.4) for each of the three wedges, as is shown in Figs. 4, $5,6,7,9$, and 10 , where the normalized pressure $p / p_{c}$ is plotted against the normalized time $t / t_{c}$. At each receiver, the partial waves are arranged in ascending order according to their minimum arrival times. Since the source and the receivers are located on the bisecting plane of each wedge, the $+k$ th and $-k$ th spherical $P$ waves arrive simultaneously.

Two source-pulses were used for the evaluation of the time records of the pressure, which are presented in Sects. 5.1, 5.2, and 5.3.

First, we assume that the source emits the Heaviside unit pulse (5.2), that is, in (2.2) we set $f(t)=t H(t)$. In this case, the partial pressure responses are (Ref. [60])

$$
p_{\mathrm{o}}=-\rho I_{\mathrm{o}}(t), \quad p_{ \pm k}=-\rho I_{ \pm k}(t)
$$

where $I_{\mathrm{o}}$ is the ray integral due to the $\phi_{\mathrm{o}}$ wave, and the first of (5.5) reduces to (5.2) since $\bar{f}(s)=1 / s^{2}$. For instance, when $R / h=5$, the normalized response $p_{\mathrm{o}} / p_{c}$ due to the pulse (5.2) sets up at time $t / t_{c}=5$, and then it remains constant at 0.2 for $t / t_{c}>5$, as is shown in Fig. 2, where $p_{\mathrm{o}} / p_{c}$ is plotted against $t / t_{c}$. The numerical integration of the ray integral $I_{\mathrm{o}}$ in (5.5) renders $p_{\mathrm{o}}$ which is in total agreement with the analytical result provided by (5.2).

Second, we assume that $f(t)$ in (2.2) is a Heaviside unit function with rounded shoulders (Refs. [49,65]), that is, $f(t)=0$ for $t \leq 0, f(t)=t^{2} / 2$ for $0<t \leq \Delta, f(t)=t^{2} / 2-(t-\Delta)^{2}$ for $0<t \leq 2 \Delta$, and 


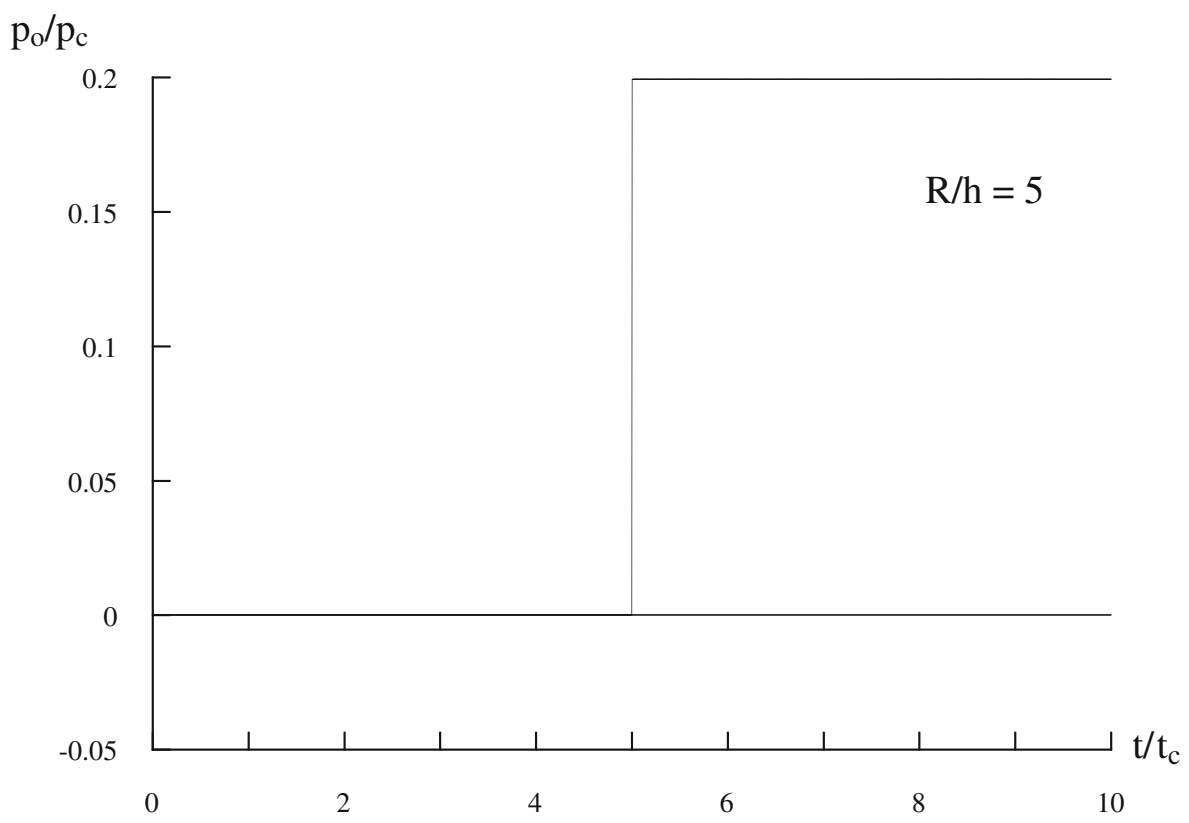

Fig. 2 Normalized source-pulse at the distance $R / h=5$ from the point source emitting the Heaviside unit pulse. The source is located in the wedge of the fluid $(c=1, \rho=1)$ at $\boldsymbol{x}_{\mathrm{o}}=\left(0,0, \cot 10^{\circ} \tan 5^{\circ}\right)$

$f(t)=\Delta^{2}$ for $t>2 \Delta$, where the rise time $2 \Delta$ is a measure of the sharpness of $f(t)$. The pressure pulse emitted by the source has thus a triangular shape (a triangular source-pulse), that is,

$$
\begin{aligned}
p_{\mathrm{o}}(t) & =0, & & t \leq R / c, \\
& =\left(p_{c} / R\right)(t-R / c), & & R / c<t \leq R / c+\Delta, \\
& =-\left(p_{c} / R\right)(t-2 \Delta-R / c), & & R / c+\Delta<t \leq R / c+2 \Delta, \\
& =0, & & t>R / c+2 \Delta .
\end{aligned}
$$

Note that, for $\Delta \rightarrow 0, f(t) / \Delta^{2}$ approaches the Heaviside unit function and $p_{0}$ becomes the Dirac delta function. The pressure responses shown in Figs. 3, 5, 7, 8b, and 10 were obtained for $\Delta / t_{c}=0.05$. For instance, when $R / h=5$, the normalized response $p_{\mathrm{o}} / p_{c}$ due to the pulse (5.6) has a triangular shape with the peak value 0.01 at $t / t_{c}=5.05$, as is shown in Fig. 3, where $p_{\mathrm{o}} / p_{c}$ is plotted against $t / t_{c}$. The numerical evaluation of $p_{\mathrm{o}} / p_{c}$ according to (4.8), where $p_{ \pm k}$ and $I_{ \pm k}$ are replaced by $p_{\mathrm{o}}$ and $I_{\mathrm{o}}$, respectively, is in total agreement with the analytical result provided by (5.6).

\subsection{Perfect wedge of fluid subject to the mixed Dirichlet-Neumann boundary conditions}

Figures 4 and 5 show the pressure records due to the image field [Eq. (5.1), $N=18$ ] at five locations, $\boldsymbol{x}_{1}, \boldsymbol{x}_{2}$, $\boldsymbol{x}_{3}, \boldsymbol{x}_{4}$, and $\boldsymbol{x}_{5}$ [Eq. (5.4)], for the perfect wedge of fluid, subject to the mixed Dirichlet-Neumann boundary conditions (2.4) and (2.5.1). The records shown in Fig. 4 were evaluated for the Heaviside unit source-pulse (5.2), and those shown in Fig. 5 for the triangular source-pulse (5.6).

In the present case of the perfect wedge of fluid, subject to the mixed Dirichlet-Neumann boundary conditions (2.4) and (2.5.1), the diffracted field is identically zero if the wedge angle $\alpha$ is an integer submultiple of $\pi / 2$ (Refs. [2,6]). Since $\alpha$ is set at $\alpha=10^{\circ}$, Figs. 4 and 5 show the exact pressure records (benchmark pressure records) due to the 3 -D field.

For the perfect wedge of fluid, subject to the mixed Dirichlet-Neumann boundary conditions (2.4) and (2.5.1), and for the Heaviside unit source-pulse (5.2), Eq. (5.5.2) reduces to

$$
p_{ \pm k}(t)=(-1)^{n}\left(p_{c} / R_{ \pm l}^{\prime}\right) H\left(t-R_{ \pm l}^{\prime} / c\right)
$$

[cf. Eq. (4.7)]. At each receiver, the numerical values of $p_{\mathrm{o}} / p_{c}$ and $p_{ \pm k} / p_{c}$ evaluated accordingly to (5.5) are in excellent agreement with the analytical values of $p_{\mathrm{o}} / p_{c}$ and $p_{ \pm k} / p_{c}$ provided by (5.2) and (5.7). 


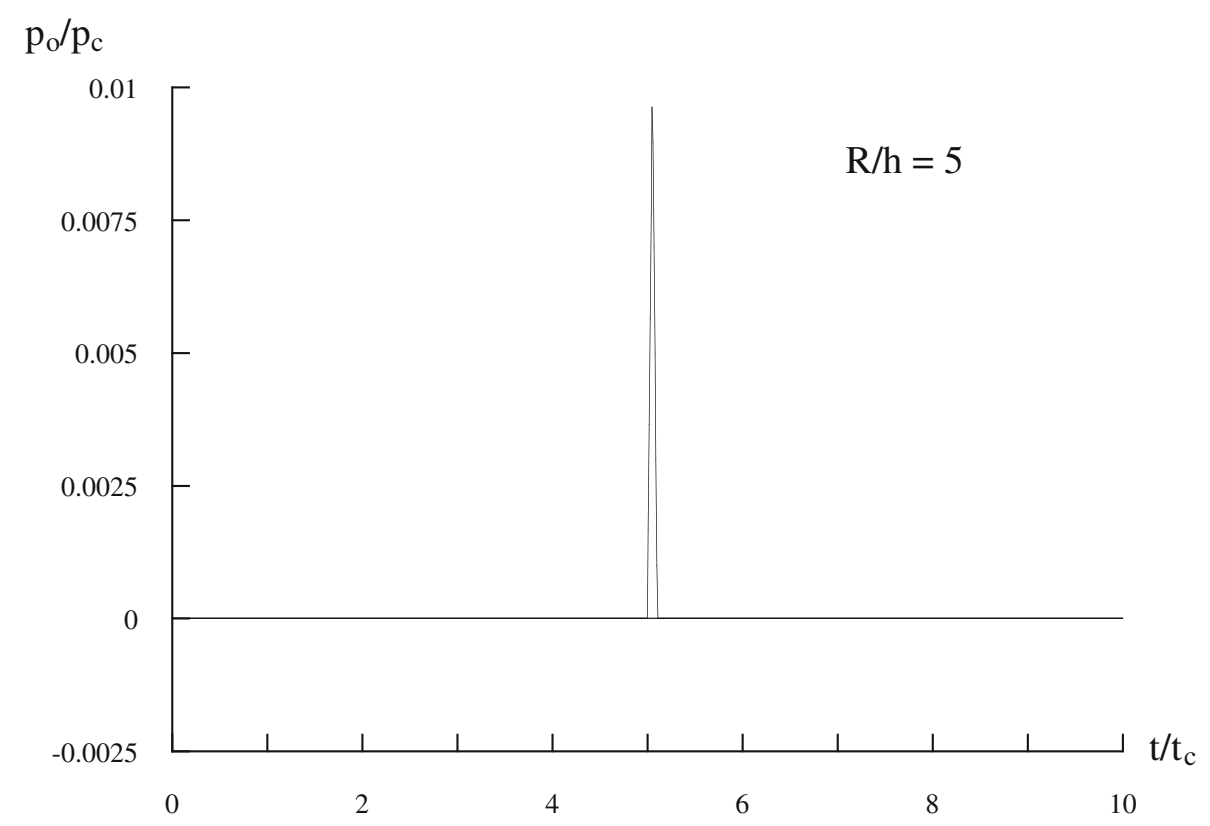

Fig. 3 Normalized source-pulse at the distance $R / h=5$ from the point source emitting the triangular pulse. The source is located in the wedge of fluid $(c=1, \rho=1)$ at $x_{\mathrm{o}}=\left(0,0, \cot 10^{\circ} \tan 5^{\circ}\right)$

Since the source and the receivers are located on the bisecting plane of the wedge, the $+k$ th and $-k$ th spherical $P$ waves arrive simultaneously, as has already been noted before; and the values of $p_{+k} / p_{c}$ and $p_{-k} / p_{c}$ are identical when $k$ is even, and the values of $p_{+k} / p_{c}$ and $p_{-k} / p_{c}$ are opposite when $k$ is odd. Thus, the contributions from the odd-order partial waves cancel out, and those from the even-order partial waves add up, so that the subsequent breaks in the pressure records shown in Fig. 4 are due to even-order multiple reflections, and each of the five pressure records shown in Fig. 5 includes 10 pulses, where some pulses following the source-pulse are more peaked than the source-pulse itself.

Each record shown in Fig. 5 can be divided into two portions: the first portion (early time response) including the contributions from pulses arriving along the direct ray paths, and the second portion (late time response) including the contributions from pulses arriving along the indirect ray paths. Note that, at $\boldsymbol{x}_{3}$ (Fig. $5 \mathrm{c}$ ), only the pulses $p_{\mathrm{o}}$ and $p_{-1}$ arrive along the direct ray paths, and all the other pulses arrive along the indirect ray paths.

Figures 4 and 5 show the effect of the receiver location on the pressure records, where the five receivers have the same radial range, but different azimuthal angles [Eq. (5.4)]. It is seen that the time interval between the arrivals of the 0th (wave emitted from the source) and the \pm 18 th spherical $P$ waves diminishes, and the pulses following the source-pulse become more peaked (Fig. 5) as the azimuthal angle increases, as is to be expected.

\subsection{Penetrable wedge of fluid with the fast-speed fluid bottom}

Figures 6 and 7 show the pressure records due to the image field [Eq. (5.1), $N=18$ ] at five locations, $\boldsymbol{x}_{1}$, $\boldsymbol{x}_{2}, \boldsymbol{x}_{3}, \boldsymbol{x}_{4}$, and $\boldsymbol{x}_{5}$ [Eq. (5.4)], for the penetrable wedge of fluid with the fast-speed fluid bottom, subject to the boundary conditions (2.4) and (2.5.2). The records shown in Fig. 6 were evaluated for the Heaviside unit source-pulse (5.2), and those shown in Fig. 7 for the triangular source-pulse (5.6).

Although the arrival times of the spherical $P$ waves are here the same as those for the case of the rigid bottom treated in Sect. 5.1, the pressure records are more complex due to the presence of the head $P$ waves, the total reflection of the spherical $P$ waves from the fast-speed fluid bottom, and the penetration of the acoustic energy into the bottom.

At each of the five locations, the pressure response begins with the arrival of the +1 st primary head $P$ wave, and the +1 st spherical $P$ wave is thus totally reflected from the fast-speed fluid bottom. This is a wide-angle reflection (a low grazing angle reflection) with the angle of incidence exceeding the critical angle 

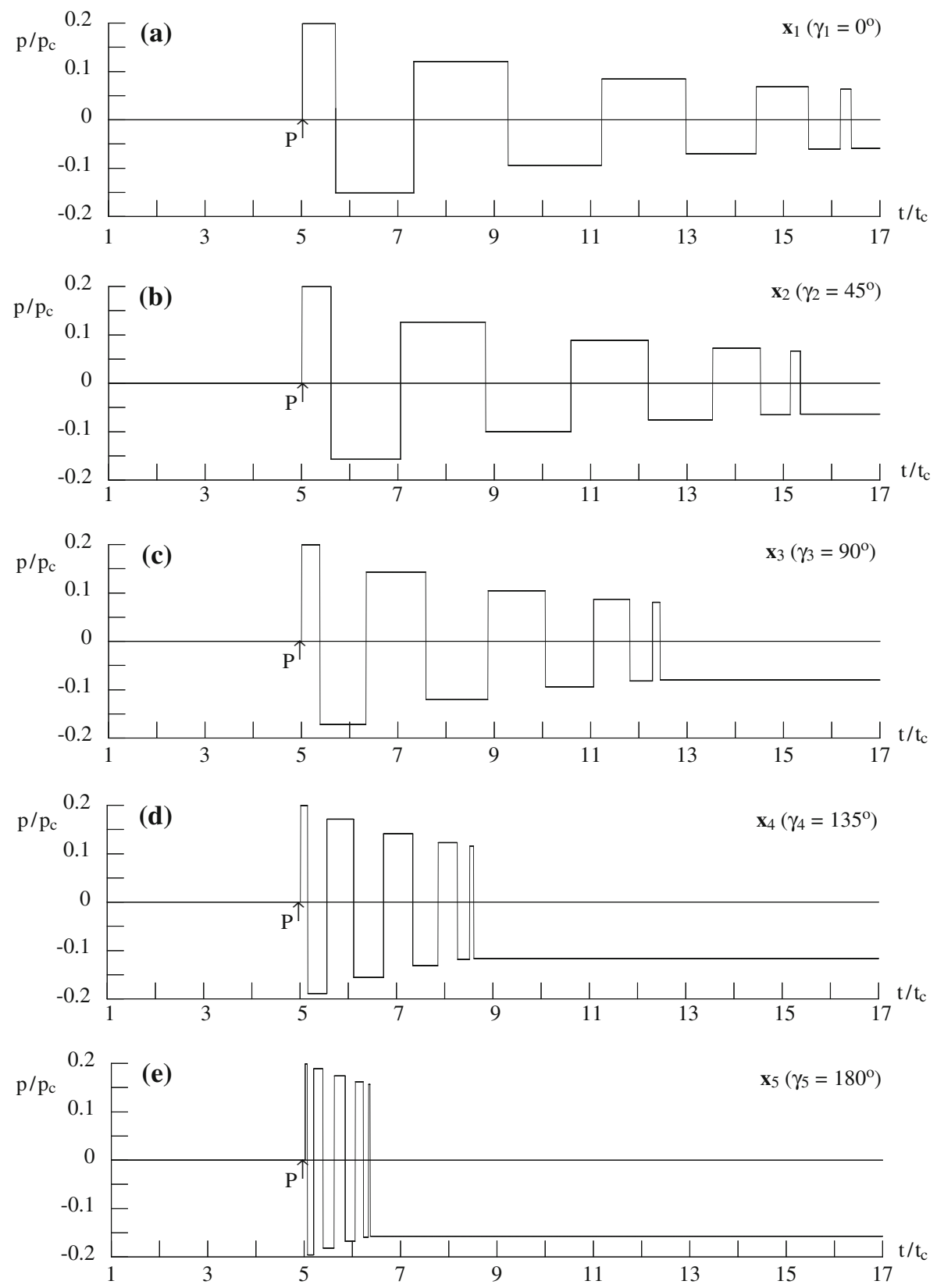

Fig. 4 Normalized pressure in the perfect wedge of fluid $(c=1, \rho=1)$ with the rigid bottom due to the point source emitting the Heaviside unit pulse; a at location $\boldsymbol{x}_{1}=\left[5,0,\left(\cot 10^{\circ}+5\right) \tan 5^{\circ}\right], \gamma_{1}=0^{\circ} ; \mathbf{b}$ at location $\boldsymbol{x}_{2}=$ $\left[5 \cos 45^{\circ}, 5 \sin 45^{\circ},\left(\cot 10^{\circ}+5 \cos 45^{\circ}\right) \tan 5^{\circ}\right], \gamma_{2}=45^{\circ} ; \mathbf{c}$ at location $\boldsymbol{x}_{3}=\left(0,5, \cot 10^{\circ} \tan 5^{\circ}\right), \gamma_{3}=90^{\circ} ; \mathbf{d}$ at location $\boldsymbol{x}_{4}=\left[-5 \sin 45^{\circ}, 5 \cos 45^{\circ},\left(\cot 10^{\circ}-5 \sin 45^{\circ}\right) \tan 5^{\circ}\right], \gamma_{4}=135^{\circ}$; e at location $\boldsymbol{x}_{5}=\left[-5,0,\left(\cot 10^{\circ}-5\right) \tan 5^{\circ}\right.$, $\gamma_{5}=180^{\circ}$. The source is located in the wedge at $x_{\mathrm{o}}=\left(0,0, \cot 10^{\circ} \tan 5^{\circ}\right)$

$\gamma_{c p}$. At each of the five locations, when the source emits the Heaviside unit pulse (5.2), the time record of the partial pulse $p_{+1}$ exhibits a singularity of the form $1 /\left(\tau-\tau_{M}\right)$ at the arrival time $\tau=\tau_{M}$ of the +1 st totally reflected spherical $P$ wave, where $\tau=t / t_{c}$ and $\tau_{M}=t_{M} / t_{c}$. For instance, at $\boldsymbol{x}_{1}$, this singularity is shown in Fig. 8a, where $p_{+1} / p_{c}$ is plotted against $t / t_{c}$. However, the record shown in Fig. 8a is finite at $\tau_{M}=5.2004$, since the numerical evaluation of the ray integral $I_{+1}$ provides data for the pulse $p_{+1}$ at a finite number of equidistant points of a fixed time interval (constant time-step), and the singularity is very narrow and sharp at $\tau_{M}=5.2004$. It was found that, when the values of the ray integral $I_{+1}$ were evaluated using the smaller time-step, only the peak value of $p_{+1} / p_{c}$ at $\tau_{M}=5.2004$ increased substantially, otherwise, the response 

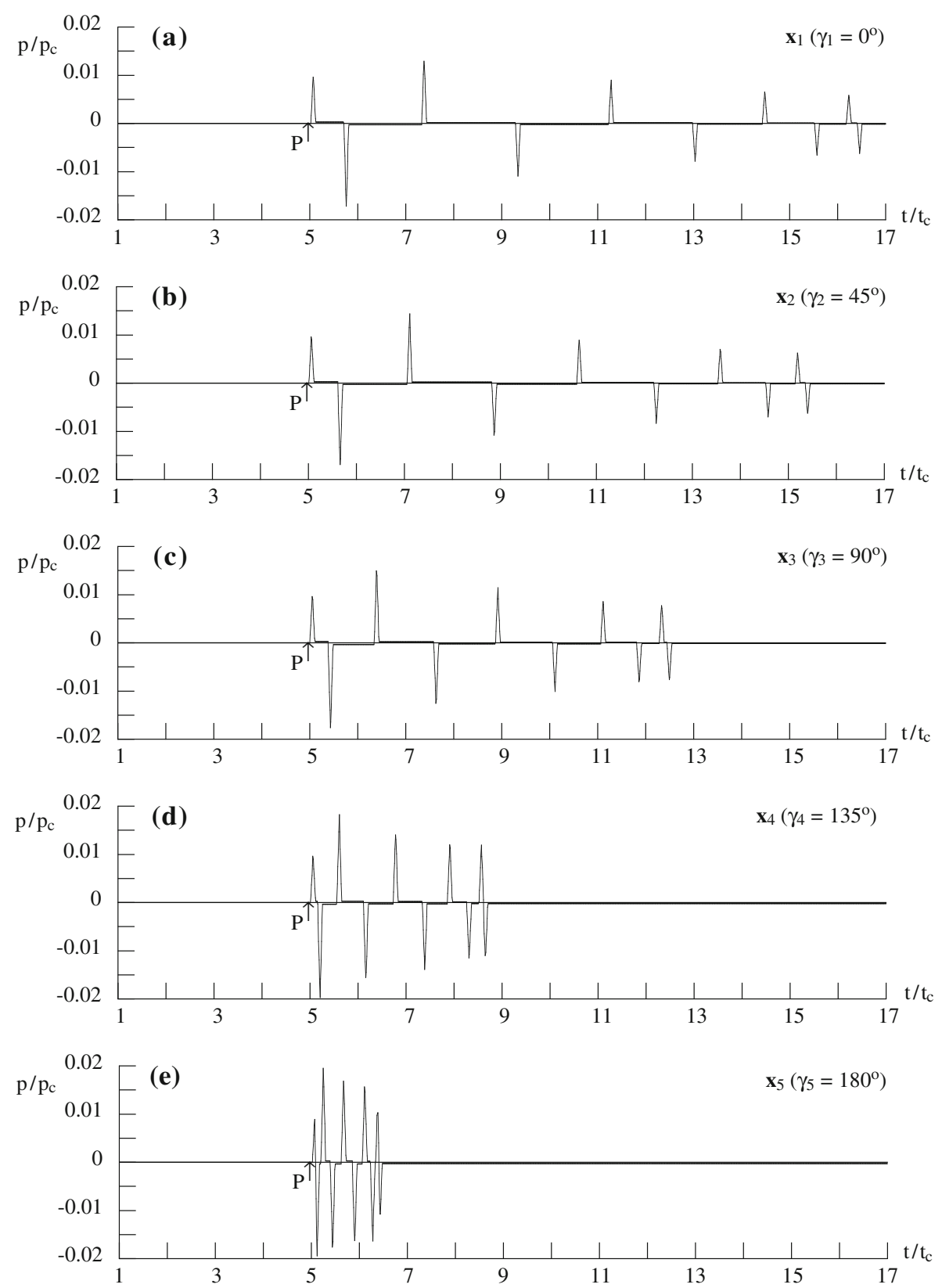

Fig. 5 Normalized pressure in the perfect wedge of fluid $(c=1, \rho=1)$ with the rigid bottom due to the point source emitting the triangular pulse; a at location $\boldsymbol{x}_{1}=\left[5,0,\left(\cot 10^{\circ}+5\right) \tan 5^{\circ}\right], \gamma_{1}=0^{\circ} ; \mathbf{b}$ at location $\boldsymbol{x}_{2}=$ $\left[5 \cos 45^{\circ}, 5 \sin 45^{\circ},\left(\cot 10^{\circ}+5 \cos 45^{\circ}\right) \tan 5^{\circ}\right], \gamma_{2}=45^{\circ} ; \mathbf{c}$ at location $\boldsymbol{x}_{3}=\left(0,5, \cot 10^{\circ} \tan 5^{\circ}\right), \gamma_{3}=90^{\circ} ; \mathbf{d}$ at location $x_{4}=\left[-5 \sin 45^{\circ}, 5 \cos 45^{\circ},\left(\cot 10^{\circ}-5 \sin 45^{\circ}\right) \tan 5^{\circ}\right], \gamma_{4}=135^{\circ}$; e at location $x_{5}=\left[-5,0,\left(\cot 10^{\circ}-5\right) \tan 5^{\circ}\right]$, $\gamma_{5}=180^{\circ}$. The source is located in the wedge at $x_{\mathrm{o}}=\left(0,0, \cot 10^{\circ} \tan 5^{\circ}\right)$

curve did not differ from that shown in Fig. 8a. A similar singularity was previously noted by Pekeris and Longman [66]. They found that, for a Heaviside unit pulse applied at a point in a horizontal fluid layer with a fast-speed fluid bottom, the partial pressure $p_{+1}$ becomes logarithmically infinite at the time of arrival of the +1 st totally reflected spherical $P$ wave.

Thus, each of the five pressure records shown in Fig. 6 exhibits the narrow and sharp peak at the arrival time of each spherical $P$ wave that undergoes one total reflection, or multiple total reflections, from the fast-speed fluid bottom. For instance, at $\boldsymbol{x}_{1}$, the record shown in Fig. 6a clearly exhibits such a peak at the arrival times 

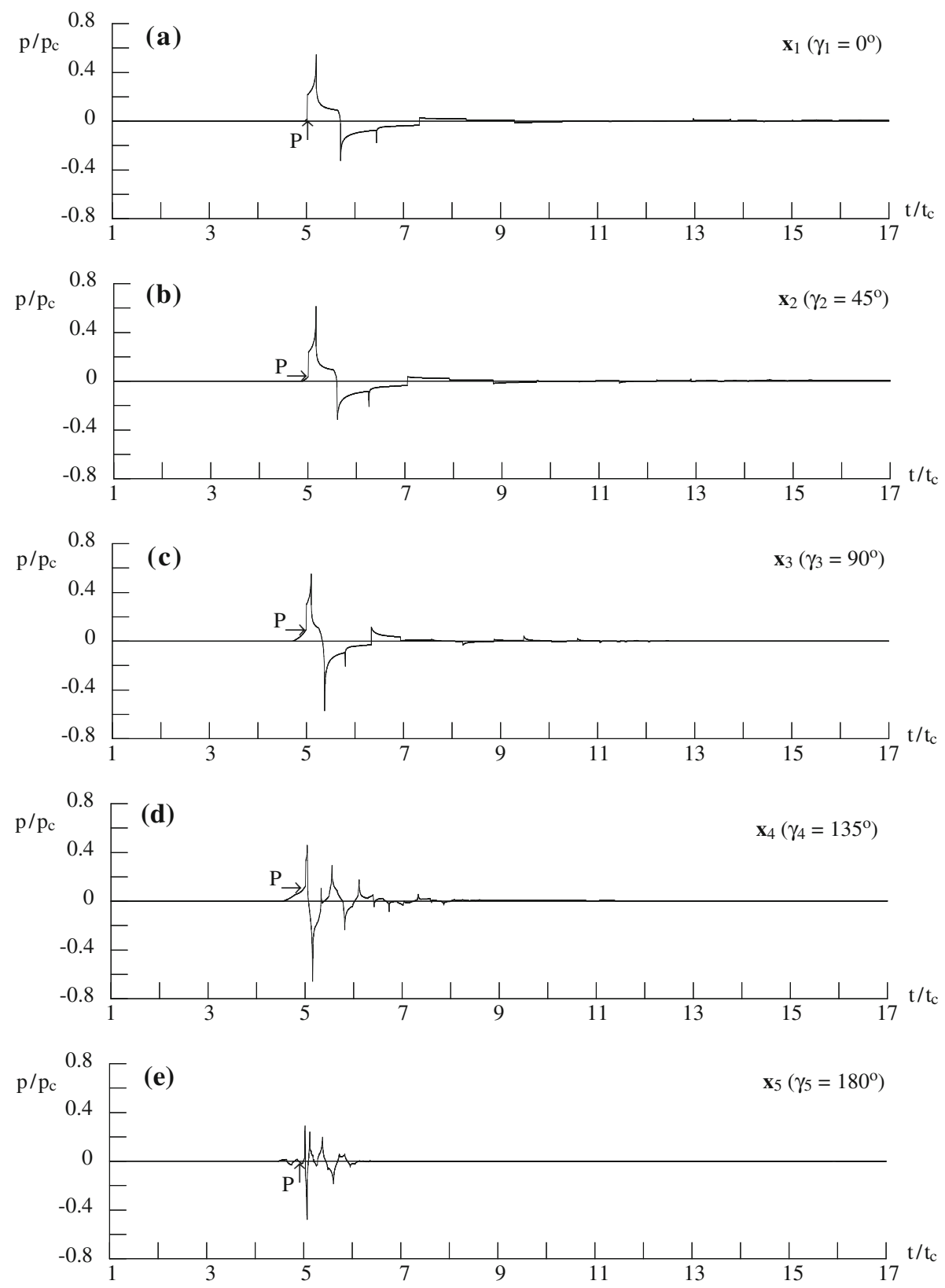

Fig. 6 Normalized pressure in the penetrable wedge of fluid $(c=1, \rho=1)$ with the fast-speed fluid bottom $\left(c_{p}=1.2, \rho_{2}=2\right)$ due to the point source emitting the Heaviside unit pulse; $\mathbf{a}$ at location $\boldsymbol{x}_{1}=\left[5,0,\left(\cot 10^{\circ}+5\right) \tan 5^{\circ}\right], \gamma_{1}=0^{\circ} ; \mathbf{b}$ at location $\boldsymbol{x}_{2}=\left[5 \cos 45^{\circ}, 5 \sin 45^{\circ},\left(\cot 10^{\circ}+5 \cos 45^{\circ}\right) \tan 5^{\circ}\right], \gamma_{2}=45^{\circ} ; \mathbf{c}$ at location $\boldsymbol{x}_{3}=\left(0,5, \cot 10^{\circ} \tan 5^{\circ}\right), \gamma_{3}=90^{\circ} ; \mathbf{d}$ at location $\boldsymbol{x}_{4}=\left[-5 \sin 45^{\circ}, 5 \cos 45^{\circ},\left(\cot 10^{\circ}-5 \sin 45^{\circ}\right) \tan 5^{\circ}\right], \gamma_{4}=135^{\circ} ; \mathbf{e}$ at $x_{5}=\left[-5,0,\left(\cot 10^{\circ}-5\right) \tan 5^{\circ}\right], \gamma_{5}=180^{\circ}$. The source is located in the wedge at $\boldsymbol{x}_{\mathrm{o}}=\left(0,0, \cot 10^{\circ} \tan 5^{\circ}\right)$

$\tau_{M}=5.2004, \tau_{M}=5.7050$, and $\tau_{M}=6.4445$ of the +1 st, -2 nd, and +3 rd totally reflected spherical $P$ waves, respectively.

The narrow and sharp peak at the arrival time of each of the totally reflected spherical $P$ waves stems from the severe time input, as represented by the Heaviside unit source-pulse (5.2). When, at each of the five locations, the convolution integrals in (4.8) were evaluated to obtain the partial pressure pulses due to the triangular source-pulse (5.6), no singular behavior was found at the arrival time of each of the totally reflected spherical $P$ waves. For instance, at $\boldsymbol{x}_{1}$, there is no singularity at $\tau_{M}=5.2004$ in the time record of the partial pulse $p_{+1}$, as is shown in Fig. 8b, where $p_{+1} / p_{c}$ is plotted against $t / t_{c}$. In this Figure, the response immediately 

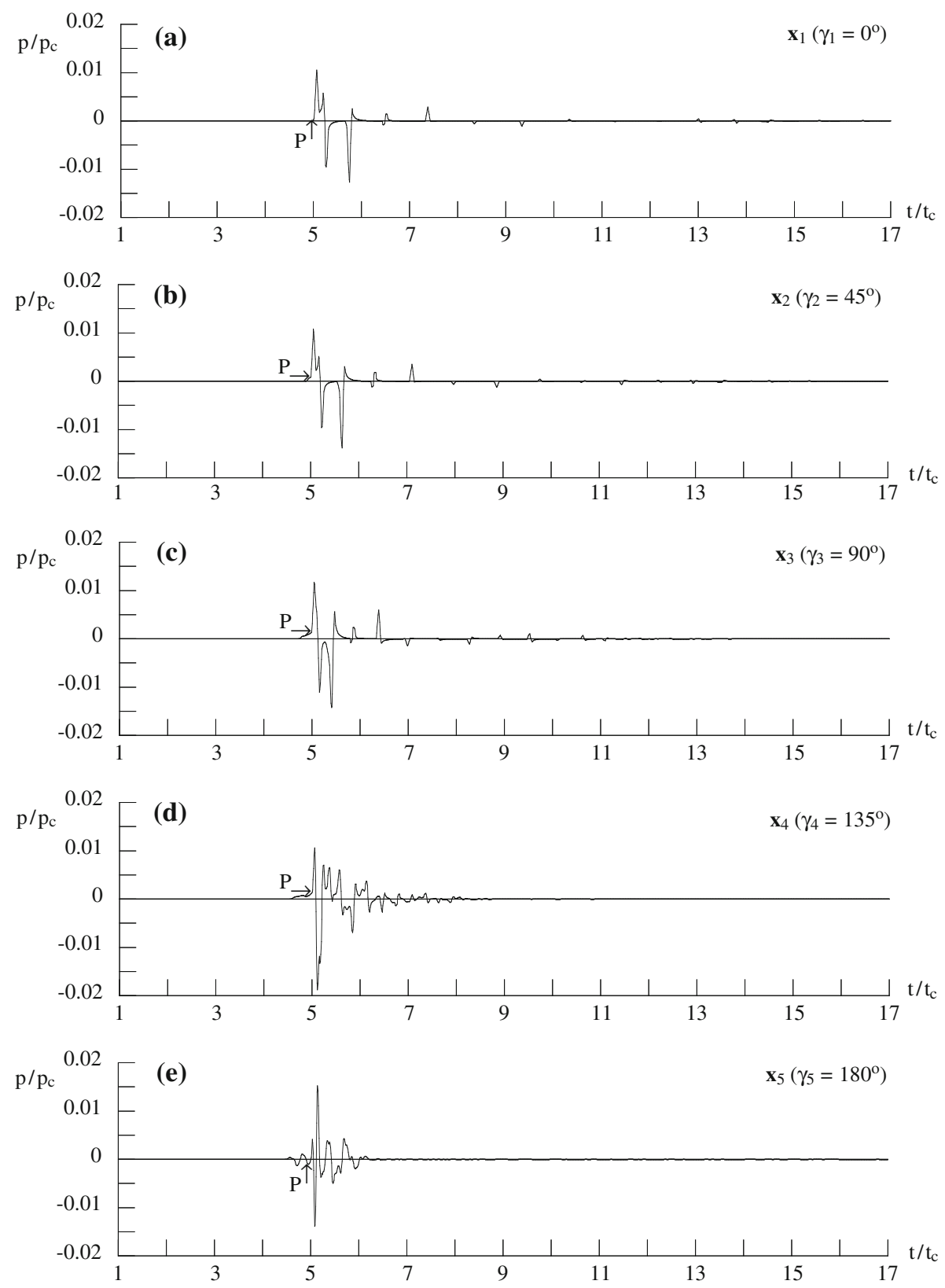

Fig. 7 Normalized pressure in the penetrable wedge of fluid $(c=1, \rho=1)$ with the fast-speed fluid bottom $\left(c_{p}=1.2, \rho_{2}=2\right)$ due to the point source emitting the triangular pulse; a at location $\boldsymbol{x}_{1}=\left[5,0,\left(\cot 10^{\circ}+5\right) \tan 5^{\circ}\right], \gamma_{1}=0^{\circ} ; \mathbf{b}$ at location $\boldsymbol{x}_{2}=\left[5 \cos 45^{\circ}, 5 \sin 45^{\circ},\left(\cot 10^{\circ}+5 \cos 45^{\circ}\right) \tan 5^{\circ}\right], \gamma_{2}=45^{\circ} ; \mathbf{c}$ at location $\boldsymbol{x}_{3}=\left(0,5, \cot 10^{\circ} \tan 5^{\circ}\right), \gamma_{3}=90^{\circ} ; \mathbf{d}$ at location $\boldsymbol{x}_{4}=\left[-5 \sin 45^{\circ}, 5 \cos 45^{\circ},\left(\cot 10^{\circ}-5 \sin 45^{\circ}\right) \tan 5^{\circ}\right], \gamma_{4}=135^{\circ}$; e at location $\boldsymbol{x}_{5}=\left[-5,0,\left(\cot 10^{\circ}-5\right) \tan 5^{\circ}\right]$, $\gamma_{5}=180^{\circ}$. The source is located in the wedge at $x_{\mathrm{o}}=\left(0,0, \cot 10^{\circ} \tan 5^{\circ}\right)$

after $\tau_{M}=5.2004$ consists of two opposite triangle-like pulses. It was found that, when the values of the ray integral $I_{+1}$ were evaluated using the smaller time-step, the evaluation of the convolution integral in (4.8) for the partial pressure $p_{+1}$ provided the same response curve as that shown in Fig. 8b. The finite slope of rise of the time input, as represented by the triangular source-pulse (5.6), thus gives the totally reflected pressure pulses with finite peak values. This observation supports that by Pekeris and Longman [66], who noted that a less sharp beginning of the source-pulse, such as one with a finite but very high slope of rise, would give a highly peaked totally reflected pulse, though with a finite maximum value.

At each of the five locations, the wave emitted from the source is preceded by the ground wave. At $x_{1}, x_{2}$, and $\boldsymbol{x}_{3}$, the ground wave includes the arrival of the +1 st primary head $P$ wave, at $\boldsymbol{x}_{4}$, the ground wave includes 


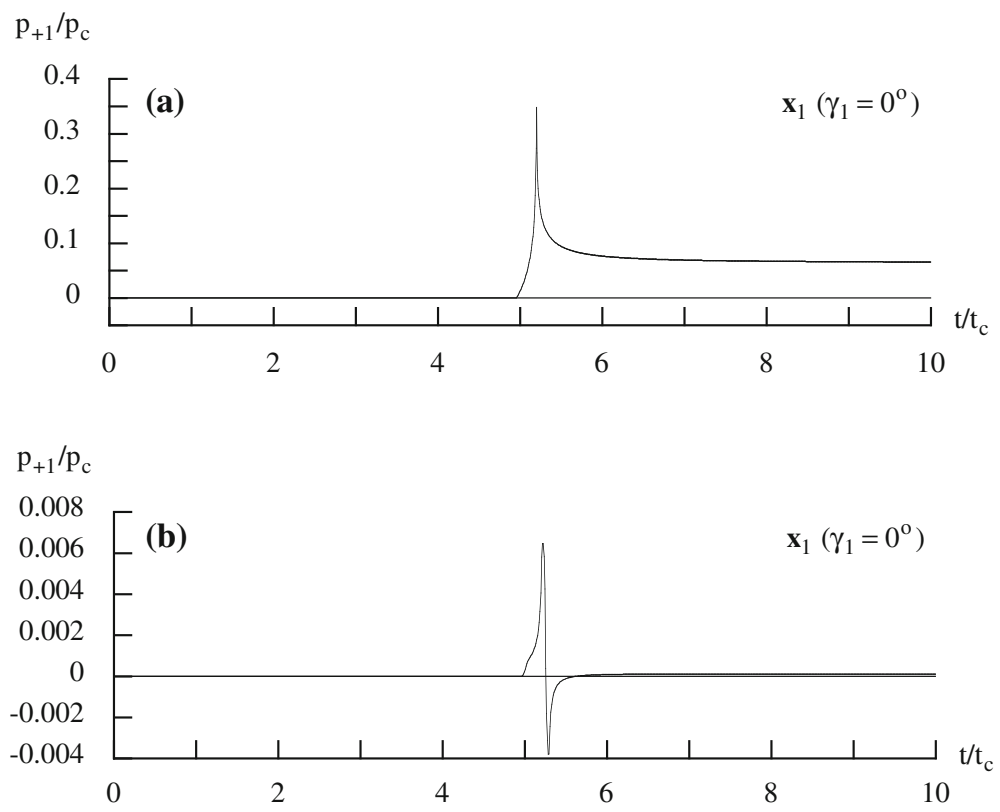

Fig. 8 Normalized partial pressure in the penetrable wedge of fluid $(c=1, \rho=1)$ with the fast-speed fluid bottom $\left(c_{p}=\right.$ $\left.1.2, \rho_{2}=2\right)$ at location $x_{1}=\left[5,0,\left(\cot 10^{\circ}+5\right) \tan 5^{\circ}\right], \gamma_{1}=0^{\circ} ;$ a due to the point source emitting the Heaviside unit pulse; b due to the point source emitting the triangular pulse. The source is located in the wedge at $x_{\mathrm{o}}=\left(0,0, \cot 10^{\circ} \tan 5^{\circ}\right)$

the arrivals of the +1 st, +2 nd, and -2 nd primary head $P$ waves, and at $x_{5}$, the ground wave includes the arrival of the $+1 \mathrm{st},+2 \mathrm{nd},+3 \mathrm{rd},+4 \mathrm{th},+5 \mathrm{th},-2 \mathrm{nd},-3 \mathrm{rd}$, and +6 th primary head $P$ waves. At $\boldsymbol{x}_{5}$, the ground wave (portion of the record for $t / t_{c}<5.0191$ ) is clearly distinguishable from the water wave (portion of the record for $t / t_{c}>5.0191$ ), as is shown in Figs. 6e and 7e, since the earliest head wave arrival is at $\tau_{ \pm l p}=4.4734$, where $\tau_{ \pm l p}=t_{ \pm l p} / t_{c}$. Note that, at each of the five locations, the water wave includes the arrivals of the multi-reflected spherical $P$ waves and the arrivals of the head $P$ waves.

The ground wave changes the peak value of the source-pulse contribution in each of the five pressure records shown in Fig. 7. At $\boldsymbol{x}_{1}$ (Fig. 7a), $\boldsymbol{x}_{2}$ (Fig. 7b), $\boldsymbol{x}_{3}$ (Fig. 7c), $\boldsymbol{x}_{4}$ (Fig. 7d), and $\boldsymbol{x}_{5}$ (Fig. 7e), this peak value is $0.0106,0.0108,0.0117,0.0106$, and 0.0042 , respectively. However, at $\boldsymbol{x}_{1}, \boldsymbol{x}_{2}, \boldsymbol{x}_{3}, \boldsymbol{x}_{4}$, and $\boldsymbol{x}_{5}$, the peak value of $p_{\mathrm{o}} / p_{c}$ due to the triangular source-pulse (5.6) alone is $0.00996,0.00998,0.01,0.00998$, and 0.00996, respectively. Thus, in the records at $\boldsymbol{x}_{1}, \boldsymbol{x}_{2}$, and $\boldsymbol{x}_{4}$ the peak value of the source-pulse contribution is slightly magnified, in the record at $\boldsymbol{x}_{3}$ this value is magnified, and in the record at $\boldsymbol{x}_{5}$ this value is reduced.

Since the critical angle for the fast-speed fluid bottom $\left(\gamma_{c p}=56.44^{\circ}\right)$ is a shallow angle, there is a large number of partial reflections off the sloping interface, which dissipate the acoustic energy into the bottom, thus reducing the peaks associated with the later arriving partial waves. This is clearly seen when the pressure records shown in Fig. 5 (rigid bottom) are compared with those shown in Fig. 7 (fast-speed fluid bottom). For instance, at $\boldsymbol{x}_{1}$ (Fig. 7a), only nine partial waves, $\phi_{0}, \phi_{ \pm 1}, \phi_{ \pm 2}, \phi_{ \pm 3}$, and $\phi_{ \pm 4}$ contribute significantly to the pressure record. Nevertheless, in the records shown in Fig. 7, some pulses following the source-pulse are still more peaked than the source-pulse itself. The effect of the receiver location on the pressure records is shown in Figs. 6 and 7, where the five receivers have the same radial range, but different azimuthal angles [Eq. (5.4)]. As the azimuthal angle increases, the time interval between the arrivals of the \pm 0 th and the \pm 18 th spherical $P$ waves diminishes, the time interval between the arrivals of the earliest and the latest primary head $P$ waves also diminishes, the pulses following the source-pulse become more peaked (Fig. 7), and the number of partial waves contributing significantly to the record thus increases.

\subsection{Penetrable wedge of fluid with the fast-speed elastic bottom}

Figures 9 and 10 show the pressure records due to the image field [Eq. (5.1), $N=18$ ] at five locations, $x_{1}$, $x_{2}, x_{3}, x_{4}$, and $x_{5}$ [Eq. (5.4)], for the penetrable wedge of fluid with the fast-speed elastic bottom, subject to 

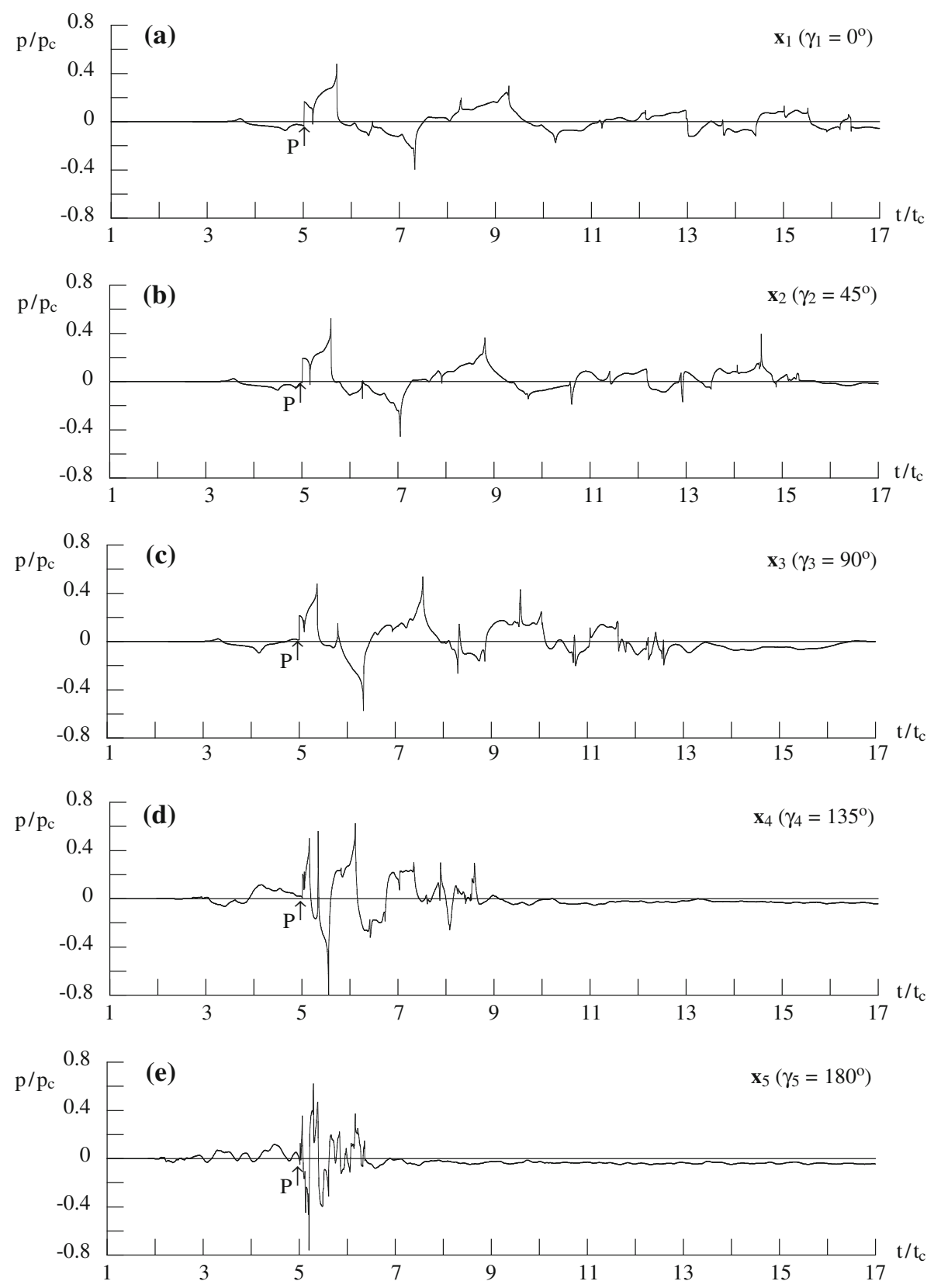

Fig. 9 Normalized pressure in the penetrable wedge of fluid $(c=1, \rho=1)$ with the fast-speed elastic bottom $\left(c_{p}=\sqrt{3} c_{s}, c_{s}=\right.$ 2.19, $\left.\rho_{2}=2.5\right)$ due to the point source emitting the Heaviside unit pulse; $\mathbf{a}$ at location $\boldsymbol{x}_{1}=\left[5,0,\left(\cot 10^{\circ}+5\right) \tan 5^{\circ}\right], \gamma_{1}=0^{\circ} ; \mathbf{b}$ at location $\boldsymbol{x}_{2}=\left[5 \cos 45^{\circ}, 5 \sin 45^{\circ},\left(\cot 10^{\circ}+5 \cos 45^{\circ}\right) \tan 5^{\circ}\right], \gamma_{2}=45^{\circ} ; \mathbf{c}$ at location $\boldsymbol{x}_{3}=\left(0,5, \cot 10^{\circ} \tan 5^{\circ}\right), \gamma_{3}=90^{\circ}$; d at location $\boldsymbol{x}_{4}=\left[-5 \sin 45^{\circ}, 5 \cos 45^{\circ},\left(\cot 10^{\circ}-5 \sin 45^{\circ}\right) \tan 5^{\circ}\right], \gamma_{4}=135^{\circ} ; \mathbf{e}$ at location $\boldsymbol{x}_{5}=\left[-5,0,\left(\cot 10^{\circ}-5\right) \tan 5^{\circ}\right]$, $\gamma_{5}=180^{\circ}$. The source is located in the wedge at $x_{\mathrm{o}}=\left(0,0, \cot 10^{\circ} \tan 5^{\circ}\right)$

the boundary conditions (2.4) and (2.5.3). The records shown in Fig. 9 were evaluated for the Heaviside unit source-pulse (5.2), and those shown in Fig. 10 for the triangular source-pulse (5.6).

The arrival times of the spherical $P$ waves are here the same as those for the case of the fast-speed fluid bottom treated in Sect. 5.2. However, because the discontinuities at the sloping interface in the $P$ wave velocity and in the density for the elastic bottom are larger than those for the fluid bottom, and (in contrast to the fluid bottom) the elastic bottom supports the $S$ waves, the pressure responses are different.

All spherical $P$ waves interacting with the bottom undergo at least one total reflection off the bottom, and (similarly as for the fast-speed fluid bottom) the pressure response at each of the five locations begins with the 

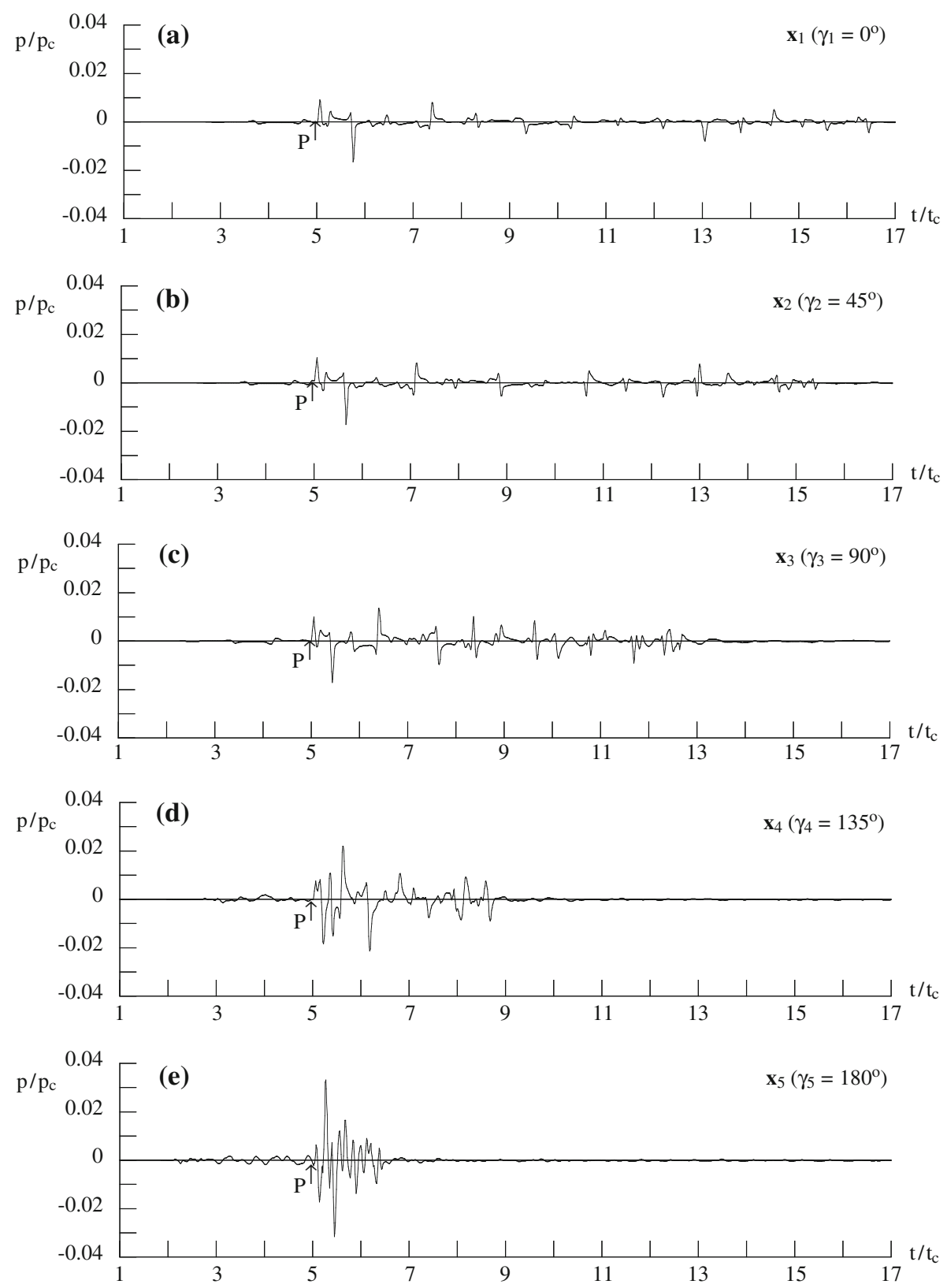

Fig. 10 Normalized pressure in the penetrable wedge of fluid $(c=1, \rho=1)$ with the fast-speed elastic bottom $\left(c_{p}=\sqrt{3} c_{s}, c_{s}=\right.$ 2.19, $\left.\rho_{2}=2.5\right)$ due to the point source emitting the triangular pulse; $\mathbf{a}$ at location $\boldsymbol{x}_{1}=\left[5,0,\left(\cot 10^{\circ}+5\right) \tan 5^{\circ}\right], \gamma_{1}=0^{\circ} ; \mathbf{b}$ at location $\boldsymbol{x}_{2}=\left[5 \cos 45^{\circ}, 5 \sin 45^{\circ},\left(\cot 10^{\circ}+5 \cos 45^{\circ}\right) \tan 5^{\circ}\right], \gamma_{2}=45^{\circ} ; \mathbf{c}$ at location $\boldsymbol{x}_{3}=\left(0,5, \cot 10^{\circ} \tan 5^{\circ}\right), \gamma_{3}=90^{\circ} ; \mathbf{d}$ at location $x_{4}=\left[-5 \sin 45^{\circ}, 5 \cos 45^{\circ},\left(\cot 10^{\circ}-5 \sin 45^{\circ}\right) \tan 5^{\circ}\right], \gamma_{4}=135^{\circ} ; \mathbf{e}$ at location $x_{5}=\left[-5,0,\left(\cot 10^{\circ}-5\right) \tan 5^{\circ}\right]$, $\gamma_{5}=180^{\circ}$. The source is located in the wedge at $x_{\mathrm{o}}=\left(0,0, \cot 10^{\circ} \tan 5^{\circ}\right)$

arrival of the +1 st (faster) primary head $P$ wave, and the time records of the partial pulses exhibit a singularity of the form $1 /\left(\tau-\tau_{M}\right)$ at the arrival time $\tau=\tau_{M}$ of the totally reflected wave, when the source emits the Heaviside unit pulse (5.2). Thus, each of the five records shown in Fig. 9 exhibits narrow and sharp peaks at the arrival times of the totally reflected waves, but these peaks are not present in each of the five records shown in Fig. 10, since these records were evaluated for the triangular source-pulse (5.6) (cf. Sect. 5.2).

At each of the five locations, the ground-wave-response of the fast-speed elastic bottom begins earlier than that of the fast-speed fluid bottom, and thus each pressure record shown in Figs. 9 and 10 separates out into distinct ground-wave and water-wave phases. At $\boldsymbol{x}_{5}$ (Figs. 9e, 10e), the ground wave is most prominent, since it includes 70 primary head wave arrivals, and the earliest of these arrivals is at $\tau_{ \pm l p}=1.8511$. 
Similarly as for the fast-speed fluid bottom, the ground wave changes the peak value of the source-pulse contribution in each of the five pressure records shown in Fig. 10. At $\boldsymbol{x}_{1}$ (Fig. 10a), $\boldsymbol{x}_{2}$ (Fig. 10b), $\boldsymbol{x}_{3}$ (Fig. 10c), $x_{4}$ (Fig. 10d), and $x_{5}$ (Fig. 10e), this peak value is 0.0084, 0.0105, 0.0101, 0.0076, and 0.0065, respectively. (The peak values of $p_{0} / p_{c}$ due to the triangular source-pulse (5.6) alone at $\boldsymbol{x}_{1}, \boldsymbol{x}_{2}, \boldsymbol{x}_{3}, \boldsymbol{x}_{4}$, and $\boldsymbol{x}_{5}$ are given in Sect. 5.2.) Thus, in the record at $x_{3}$ the peak value of the source-pulse contribution is almost not changed, in the record $\boldsymbol{x}_{2}$ this value is slightly magnified, and in the records at $\boldsymbol{x}_{1}, \boldsymbol{x}_{4}$, and $\boldsymbol{x}_{5}$ this value is reduced.

In contrast to the case of the fast-speed fluid bottom, the two critical angles for the fast-speed elastic bottom $\left(\gamma_{c s}=27.17^{\circ}\right.$ and $\left.\gamma_{c p}=15.29^{\circ}\right)$ are steep. Hence, there are less partial reflections off the sloping interface, so that the penetration of the acoustic energy into the fast-speed elastic bottom is much weaker, and thus even the later arriving partial waves undergoing many bottom reflections make significant contributions to the pressure responses (Fig. 10). Note that, at $\boldsymbol{x}_{1}$, the response curve shown in Fig. 9a clearly exhibits the break at the arrival time of the wave emitted from the source and the peaks at the arrival times of all (even-order and odd-order) spherical $P$ waves contributing to the record, with the exception of the peak at $\tau_{M}=16.3484$ due to the \pm 17 th spherical $P$ waves, since this arrival time is very close to that at $\tau_{M}=16.4050$ due to the \pm 18 th spherical $P$ waves. Figures 9 and 10 demonstrate the dependence of the pressure responses on the receiver position, where the five receivers have the same radial range, but different azimuthal angles [Eq. (5.4)]. As the azimuthal angle increases, the duration of the ground-wave phase also increases, but the duration of the water-wave phase decreases, and the pulses following the source-pulse become more peaked (Fig. 10). At $\boldsymbol{x}_{4}$, two pulses in Fig. 10d (fast-speed elastic bottom) are more peaked than their two counterparts (pulses due to the \pm 4 th and \pm 6 th spherical $P$ waves) in Fig. $5 \mathrm{~d}$ (rigid bottom), and, at $\boldsymbol{x}_{5}$, the amplification of the two pulses is even stronger (Figs. 5e, 10e). This effect is probably due to the constructive interference, since the fast-speed elastic bottom has a tendency to return the acoustic energy toward the upper layer (wedge of fluid) (cf. Ref. [66]).

\section{Conclusions}

As mentioned in Sect. 1, the 3-D field generated by a point source in a wedge consists of two components: the diffracted field arising from scattering at the apex of the wedge, and the image field expressed by a sum of partial waves including the wave radiated from the source plus a finite number of waves reflected from the wedge boundaries.

The purpose of this paper was to present the generalized ray solution for the 3-D pressure field in a rigidbottom, fast-speed fluid-bottom, and fast-speed elastic-bottom wedge. The contribution from the diffracted field is not included in the solution, but nevertheless the solution is exact and complete for the image field because no approximations were introduced into the mathematical formulation of the problem, and the ray integrals are evaluated exactly by applying the Cagniard-de Hoop method; thus the contribution of the entire image field is included for the complete received signal, as opposed to the method of plane wave approximation (geometrical acoustics approximation) evaluating the specular component of the field only (Refs. $[18,67])$.

Since the wedge angle $\alpha$ was set at $\alpha=10^{\circ}$, the complete image field included 37 partial waves, and the diffracted field was identically zero in the rigid-bottom wedge. The source and five receivers were placed on the bisecting plane of the wedge, each receiver had the same radial range in the horizontal from the source, but different orientation relative to the source, measured by the azimuthal angle assuming values $0^{\circ}$ (down-slope), $45^{\circ}$ (obliquely down-slope), $90^{\circ}$ (cross-slope), $135^{\circ}$ (obliquely up-slope), and $180^{\circ}$ (up-slope). Time records of the normalized pressure due to the complete image field were evaluated at the five receivers for two transient source-pulses: the Heaviside unit pulse and the triangular pulse.

These pressure records provided information about the 3-D image field in the wedge. For each of the three bottoms, the time interval between the first arrival and the two ultimate arrivals diminished, and the pulses following the source-pulse became more peaked, as the azimuthal angle of the receiver increased.

In the rigid-bottom wedge (perfect wedge), there was no penetration of the acoustic energy into the bottom, and all pressure pulses arriving along indirect paths were significant at each of the five receivers. Some pulses following the source-pulse were more peaked than the source-pulse itself because the source and the receivers were located on the bisector of the wedge, so that the odd-order pulses canceled out and the even-order pulses added up.

In the fluid-bottom wedge, at each of the five receivers, the spherical $P$ wave emitted from the source was preceded by the ground wave including head wave arrivals whose amplitudes were much weaker than those of the spherical $P$ waves (waterborne waves). The amplitudes of the later arriving (that is, higher-order) 
spherical $P$ waves were severely reduced due to partial reflections off the sloping interface, which dissipated the acoustic energy into the bottom. The inspection of the pressure records indicated that the pulses arriving along indirect paths were insignificant at each of the five receivers for the fluid bottom with shallow critical angle $\left(\gamma_{c p}=56.44^{\circ}\right)$.

In the elastic-bottom wedge, the two critical angles $\left(\gamma_{c s}=27.17^{\circ}\right.$ and $\left.\gamma_{c p}=15.29^{\circ}\right)$ were steep. Hence, there were less partial reflections off the sloping interface, so that the penetration of the acoustic energy into the bottom was much weaker, and the pulses arriving along indirect paths made significant contributions to the pressure records. At each of the five receivers, the ground-wave-response began earlier than that in the fluid-bottom wedge, and thus the pressure record separated out into distinct ground-wave and water-wave phases.

Acknowledgements Open access funding provided by TU Wien (TUW). The research work reported in this paper was made from 1998 to 2013 by Prof. Yih-Hsing Pao, Prof. Chi-Fang Chen, and Dr. Piotr Borejko; in June, 2013, Prof. Yih-Hsing Pao passed away; from then on the work was continued until 2017 by Prof. Chi-Fang Chen and Dr. Piotr Borejko. The work was funded in 1998-1999 by the National Science Council (currently the Ministry of Science and Technology) of the Republic of China when Dr. Piotr Borejko was employed for two years as a guest researcher and worked closely with Prof. Yih-Hsing Pao and Prof. Chi-Fang Chen at the National Taiwan University (NTU), Taipei, Taiwan, Republic of China. The work was also funded (short visits) in 2009, 2013, 2014, and 2017 by the TU Wien, Vienna, Austria, when Prof. Chi-Fang Chen visited Dr. Piotr Borejko at the TU Wien; in 2005 and 2011 by the NTU when Dr. Piotr Borejko visited Prof. Yih-Hsing Pao and Prof. Chi-Fang Chen at the NTU; and again in 2015 by the NTU when Dr. Piotr Borejko visited Prof. Chi-Fang Chen at the NTU. The authors are pleased to acknowledge a number of improvements suggested by anonymous reviewers.

Open Access This article is distributed under the terms of the Creative Commons Attribution 4.0 International License (http:// creativecommons.org/licenses/by/4.0/), which permits unrestricted use, distribution, and reproduction in any medium, provided you give appropriate credit to the original author(s) and the source, provide a link to the Creative Commons license, and indicate if changes were made.

\section{References}

1. Sommerfeld, A.: Partial Differential Equations in Physics. Academic Press, New York (1949)

2. Biot, M.A., Tolstoy, I.: Formulation of wave propagation in infinite media by normal coordinates with application to diffraction. J. Acoust. Soc. Am. 29, 381-391 (1957)

3. Tolstoy, I.: Wave Propagation. McGraw-Hill, New York (1973)

4. Buckingham, M.J.: Acoustic propagation in a wedge-shaped ocean with perfectly reflecting boundaries. In: Felsen, L.B. (ed.) Hybrid Formulation of Wave Propagation and Scattering, pp. 77-105. Martinus Nijhoff, Dordrecht (1984)

5. Buckingham, M.J.: Acoustic propagation in a wedge-shaped ocean with perfectly reflecting boundaries. NRL Rep. 8793 (1984)

6. Buckingham, M.J.: Theory of acoustic radiation in corners with homogeneous and mixed perfectly reflecting boundaries. J. Acoust. Soc. Am. 86, 2273-2291 (1989)

7. Keller, J.B.: Surface waves on water of nonuniform depth. J. Fluid Mech. 4, 607-614 (1958)

8. Kuznetsov, V.K.: A new method for solving the problem of the sound field in a fluid wedge. Sov. Phys. Acoust. 5, 171-176 (1959)

9. Weston, D.E.: Horizontal refraction in a three-dimensional medium of variable stratification. Proc. Phys. Soc. 78, 46-52 (1961)

10. Pierce, A.D.: Extension of the method of normal modes to sound propagation in an almost stratified medium. J. Acoust. Soc. Am. 37, 19-27 (1965)

11. Kuznetsov, V.K.: The refraction of normal modes in a wedge. Vestn. Mosk. Univ. Fiz. 22, 14-21 (1967)

12. Harrison, C.H.: Acoustic shadow zones in the horizontal plane. J. Acoust. Soc. Am. 65, 56-61 (1979)

13. Westwood, E.K.: Ray model solutions to the benchmark wedge problems. J. Acoust. Soc. Am. 87, 1539-1545 (1990)

14. Bradley, D.L., Hudimac, A.A.: The propagation of sound in a wedge-shaped shallow water duct. Naval Ord. Lab. Rep. NOLTR, 70-235 (1970)

15. Graves, R.D., Nagl, A., Überall, H., Zarur, G.L.: Range-dependent normal modes in underwater sound propagation: application to the wedge-shaped ocean. J. Acoust. Soc. Am. 58, 1171-1177 (1975)

16. Buckingham, M.J., Tolstoy, A.: An analytical solution for benchmark problem 1: the "ideal" wedge. J. Acoust. Soc. Am. 87, 1511-1513 (1990)

17. Buckingham, M.J.: Theory of three-dimensional acoustic propagation in a wedgelike ocean with a penetrable bottom. J. Acoust. Soc. Am. 82, 198-210 (1987)

18. Frisk, G.V.: Ocean and Seabed Acoustics: A Theory of Wave Propagation. Prentice-Hall, Englewood Cliffs (1994)

19. Stickler, D.C.: Normal-mode program with both the discrete and branch-line contributions. J. Acoust. Soc. Am. 57, 856-861 (1975)

20. Chapman, D.M.F., Ward, P.D.: The normal-mode theory of air-to-water sound transmission in the ocean. J. Acoust. Soc. Am. 87, 601-618 (1990)

21. Weinberg, H., Burridge, R.: Horizontal ray theory for ocean acoustics. J. Acoust. Soc. Am. 55, 63-79 (1974)

22. Eby, R.K., Williams Jr., A.O., Ryan, R.P., Tamarkin, P.: Study of acoustic propagation in a two-layered model. J. Acoust. Soc. Am. 32, 88-99 (1960) 
23. Milder, D.M.: Ray and wave invariants for SOFAR channel propagation. J. Acoust. Soc. Am. 46, 1259-1263 (1969)

24. Pierce, A.D.: Guided mode disappearance during upslope propagation in variable depth shallow water overlying a fluid bottom. J. Acoust. Soc. Am. 72, 523-531 (1982)

25. Pierce, A.D.: Augmented adiabatic mode theory for upslope propagation from a point source in variable-depth shallow water overlying a fluid bottom. J. Acoust. Soc. Am. 74, 1837-1847 (1983)

26. Jensen, F.B., Kuperman, W.A.: Sound propagation in a wedge-shaped ocean with a penetrable bottom. J. Acoust. Soc. Am. 67, 1564-1566 (1980)

27. Tindle, C.T., Hobaek, H., Muir, T.G.: Downslope propagation of normal modes in a shallow water wedge. J. Acoust. Soc. Am. 81, 275-286 (1987)

28. Tindle, C.T., Hobaek, H., Muir, T.G.: Normal mode filtering for downslope propagation in a shallow water wedge. J. Acoust. Soc. Am. 81, 287-294 (1987)

29. Ingenito, F.: Excitation, propagation and attenuation of acoustic normal modes in shallow water. In: Sound Propagation in Shallow Water, SACLANTCEN Conference Proceedings No. 14, pp. 69-78 (1974)

30. Wolf, S.N.: Measurements of normal mode amplitude function in a nearly stratified medium. In: Ocean Acoustic Modeling, Proceedings of SACLANTCEN Conference (1975)

31. Jensen, F.B., Kuperman, W.A.: Range-dependent bottom-limited propagation modeling with the parabolic equation. In: Bottom Interacting Ocean Acoustics. Plenum Press, New York (1980)

32. Tindle, C.T., Bold, G.E.J.: Improved ray calculations in shallow water. J. Acoust. Soc. Am. 70, 813-819 (1981)

33. Tindle, C.T.: Ray calculations with beam displacement. J. Acoust. Soc. Am. 73, 1581-1586 (1983)

34. Westwood, E.K., Tindle, C.T.: Shallow water time-series simulation using ray theory. J. Acoust. Soc. Am. 81, 1752-1761 (1987)

35. Westwood, E.K.: Broadband modeling of the three-dimensional penetrable wedge. J. Acoust. Soc. Am. 92, 2212-2222 (1992)

36. Westwood, E.K.: Ray methods for flat and sloping shallow-water wave-guides. J. Acoust. Soc. Am. 85, 1885-1894 (1989)

37. Sturm, F.: Numerical study of broadband sound pulse propagation in three-dimensional oceanic wave-guides. J. Acoust. Soc. Am. 117, 1058-1079 (2005)

38. Sturm, F., Korakas, A.: Comparisons of laboratory scale measurements of three-dimensional acoustic propagation with solutions by a parabolic equation model. J. Acoust. Soc. Am. 133, 108-118 (2013)

39. Korakas, A., Sturm, F., Sessarego, J.-P., Ferrand, D.: Scaled model experiment of long-range across-slope propagation in a penetrable wedge. J. Acoust. Soc. Am. 126, EL22-EL27 (2009)

40. Jensen, F.B., Tindle, C.T.: Numerical modeling results for mode propagation in a wedge. J. Acoust. Soc. Am. 82, 211-216 (1987)

41. Wood, A.B.: Model experiments on sound propagation in shallow seas. J. Acoust. Soc. Am. 31, 1213-1235 (1959)

42. Doolittle, R., Tolstoy, A., Buckingham, M.J.: Experimental confirmation of horizontal refraction of cw acoustic radiation from a point source in a wedge-shaped ocean environment. J. Acoust. Soc. Am. 83, 2117-2125 (1988)

43. Glegg, S.A.L., Yoon, J.R.: Experimental measurements of three-dimensional propagation in a wedge-shaped ocean with pressure-release boundary conditions. J. Acoust. Soc. Am. 87, 101-105 (1990)

44. Glegg, S.A.L., Deane, G.B., House, I.G.: Comparison between theory and model scale measurements of three-dimensional sound propagation in shear supporting penetrable wedge. J. Acoust. Soc. Am. 94, 2334-2342 (1993)

45. Deane, G.B., Buckingham, M.J.: An analysis of the three-dimensional sound field in a penetrable wedge with a stratified fluid or elastic basement. J. Acoust. Soc. Am. 93, 1319-1328 (1993)

46. Heaney, K.D., Campbell, R.L., Murray, J.J.: Comparison of hybrid three-dimensional modeling with measurements on the continental shelf. J. Acoust. Soc. Am. 131, 1680-1688 (2012)

47. Heaney, K.D., Murray, J.J.: Measurements of three-dimensional propagation in a continental shelf environment. J. Acoust. Soc. Am. 125, 1394-1402 (2009)

48. Ballard, M.S.: Modeling three-dimensional propagation in a continental shelf environment. J. Acoust. Soc. Am. 131, 19691977 (2012)

49. Pao, Y.H., Gajewski, R.R.: The generalized ray theory and transient responses of layered elastic solids. In: Mason, W.P., Thurston, R.N. (eds.) Physical Acoustics, pp. 183-265. Academic Press, New York (1977)

50. Pao, Y.H.: Elastic waves in solids. ASME J. Appl. Mech. 50, 1152-1164 (1983)

51. Kennett, B.L.N.: Seismic Wave Propagation in Stratified Media. Cambridge University Press, Cambridge (1983)

52. Pao, Y.H., Ziegler, F.: Transient SH-waves in a wedge-shaped layer. Geophys. J. R. Astron. Soc. 71, 57-77 (1982)

53. Ziegler, F., Pao, Y.H.: Transient elastic waves in a wedge-shaped layer. Acta Mech. 52, 133-163 (1984)

54. Ziegler, F., Pao, Y.H.: Theory of generalized rays for SH-waves in dipping layers. Wave Motion 7, 1-24 (1985)

55. Ziegler, F., Pao, Y.H., Wang, Y.S.: Transient SH-waves in dipping layers: the buried line-source problem. J. Geophys. 57, 23-32 (1985)

56. Ziegler, F., Pao, Y.H., Wang, Y.S.: Generalized ray-integral representation of transient SH-waves in multiply layered halfspace with dipping structure. Acta Mech. 56, 1-15 (1985)

57. Borejko, P.: Reflection and transmission coefficients for three-dimensional plane waves in elastic media. Wave Motion 24, 371-393 (1996)

58. Borejko, P., Ziegler, F.: Pulsed asymmetric point force loading of a layered half-space. In: Guran, A., Böstrom, A., Leroy, O., Maze, G. (eds.) Series on Stability, Vibration and Control of Systems, Series B, Vol. 5, Acoustic Interactions with Submerged Elastic Structures Part IV: Nondestructive Testing, Acoustic Wave Propagation and Scattering, pp. 307-388. World Scientific, Singapore (2002)

59. Pao, Y.H., Ziegler, F., Wang, Y.S.: Acoustic waves generated by a point source in a sloping fluid layer. J. Acoust. Soc. Am. 85, 1414-1426 (1989)

60. Borejko, P., Chen, C.F., Pao, Y.H.: Application of the method of generalized rays to acoustic waves in a liquid wedge over elastic bottom. J. Comput. Acoust. 9, 41-68 (2001)

61. de Hoop, A.T.: A modification of Cagniard's method for solving seismic pulse problems. Appl. Sci. Res. B 8, 349-356 (1960) 
62. Cagniard, L.: Reflection and Refraction of Progressive Seismic Waves (McGraw-Hill, New York, translated and revised by E. A. Flinn and C. H. Dix) (1962)

63. Cerveny, V., Ravindra, R.: Theory of Seismic Head Waves. University of Toronto Press, Toronto (1971)

64. Aki, K., Richards, P.G.: Quantitative Seismology: Theory and Methods, vol. 1. Freeman, San Francisco (1980)

65. Pekeris, C.L., Alterman, Z., Abramovici, F., Jarosch, H.: Propagation of a compressional pulse in a layered solid. Rev. Geophys. 3, 25-47 (1965)

66. Pekeris, C.L., Longman, I.M.: Ray theory solution of the problem of propagation of explosive sound in a layered liquid. J. Acoust. Soc. Am. 30, 323-328 (1958)

67. Pierce, A.D.: Acoustics: An Introduction to its Physical Principles and Applications. Acoustical Society of America, New York (1989) 